\title{
3 taedia sustineas - zeitgenössische Reflexionen über Schrift im öffentlichen Raum
}

Der Umgang mit Geschriebenem, aber auch Aspekte der Präsenz und Materialität von Schrift im öffentlichen Raum finden Reflexe in verschiedenen Medien, die sich als Spuren des zeitgenössischen Diskurses erhalten haben. In diesem Kapitel sollen zunächst Äußerungen, die sich in fiktionalen und nicht-fiktionalen Texten antiker Autoren finden, vorgestellt und ausgewertet werden. Diese Textausschnitte bieten Aussagen über Schreiber, Rezipienten und Wertungen von Schrift sowie über mögliche Anbringungsorte, Schreibanlässe und verwendete Schreibgeräte. Als zweite Gruppe von Zeugnissen werden Inschriften aus Pompeji und Herculaneum besprochen, die in besonderem Maße auf andere Texte oder sich selbst als Text oder Medium verweisen oder Vorgänge von Produktion und Rezeption explizit thematisieren. Im dritten Abschnitt werden bildliche Darstellungen aus den beiden Städten behandelt, die innerhalb der Bilder, also im Modus der Darstellung, auch Schrift enthalten. Diese ermöglichen es aufgrund der komplexen Darstellung und Bildkomposition, Rückschlüsse auf die Beurteilung optischer Qualitäten und der kontextuellen Verortung von Schrift zu ziehen.

Alle drei Zeugnisgruppen können als „Metatexte“ aufgefasst werden. ${ }^{453}$ Unter Metatexten sollen hier zunächst alle Texte verstanden werden, die Aussagen über Geschriebenes treffen und in denen, wie Jan Christian Gertz, Frank Krabbes und Eva Noller betonen, „Diskurse über Schriftlichkeit sichtbar [werden], deren Analyse die Herausarbeitung sowie den diachronen und inter-/intrakulturellen Vergleich jener Aspekte gestattet, die zeitgenössische Autoren und Erzähler mit dem Geschriebenen verbanden“. ${ }^{454}$ Wenn in diesem Zusammenhang auch bildliche Darstellungen in die Überlegungen miteinbezogen werden, so ist damit nicht der Versuch verbunden, Bilder wie Texte zu lesen, sondern vielmehr, zu fragen, inwieweit Schrift auch bildlichen

453 Vgl. Wolf 2008, 494-495.

454 Siehe dazu Gertz et al. 2015. Das Potential der Metatexte wird dort folgendermaßen charakterisiert: „Mithin ermöglicht die Metatextanalyse also die Rekonstruktion derjenigen Aspekte eines Diskurses über Schriftlichkeit, die mit der effektiven Materialität und Präsenz des Geschriebenen und ihrem Wirken auf das menschliche Handeln durch die Zeitgenossen in Verbindung stehen.“. Gertz, Krabbes und Noller weisen jedoch auch auf die Einschränkungen hin: „Folgt man der forschungstheoretischen Prämisse, dass dem Geschriebenem kein ihm quasi substantiell eigener Sinngehalt innewohnt, sondern Bedeutungszuschreibungen vielmehr Resultate historisch und sozio-kulturell spezifischer Rezeptionsakte sind, ergibt sich die Konsequenz, dass der eigentlichen Metatextanalyse die Rekonstruktion des historischen und sozio-kulturellen Kontextes sowie der konkreten situativen Verortungen der rezipierenden Subjekte vorausgehen muss.“ Zur Bedeutung von Metatexten vgl. auch: Hilgert 2010, 94-95.

Ә Open Access. ( 2021 Fanny Opdenhoff, publiziert von De Gruyter. (c) BY-NC-ND Dieses Werk ist lizenziert unter der Creative Commons Attribution-NonCommercial-NoDerivatives 4.0 Lizenz. 
Charakter haben konnte, und zu beschreiben, welche Merkmale von Schrift im Rahmen solcher Darstellungen zum Tragen kamen. ${ }^{455}$

Konkret sollen bei der Analyse der Metatexte die folgenden Fragen gestellt werden, um so die Ergebnisse aus der Untersuchung der Inschriften zu ergänzen: Welche Personen sind an der Anfertigung der Inschriften beteiligt? An welchen Orten werden sie angebracht? Welche Materialien werden verwendet? Um welche Themen und Inhalte geht es in den Inschriften? Was sind die Anlässe? Welche Rezeptionspraktiken werden geschildert oder erwähnt und wer ist daran beteiligt? Wie werden Schreib- und Lesepraktiken sowie das Erscheinungsbild und die Präsenz der In- und Aufschriften beurteilt? Insbesondere bei der Beantwortung der letztgenannten Frage gilt es, die Aussageabsichten der Schriftquellen und die erzählerische Funktion der erwähnten Texte und Schriften im Blick zu behalten.

\subsection{Literarische Quellen}

Im Folgenden sind Textstellen, in denen explizit auf den Schriftgebrauch im öffentlichen Raum und insbesondere Graffiti Bezug genommen wird, aufgeführt. Sie werden jeweils mit einer kurzen inhaltlichen Einordnung in den Erzählzusammenhang des Textes, dem sie entnommen sind, eingeführt. ${ }^{456}$ Die Textstellen stammen aus einem Zeitraum von ca. 250 Jahren und sind soweit möglich in chronologischer Reihenfolge angegeben.

\subsubsection{Textstellen}

(1) Plaut. Merc. 405-409 (nach 211 v. Chr.): quia illa forma matrem familias / flagitium sit sei sequatur; quando incedat per vias, / contemplent, conspiciant omnes, nutent, nictent, sibilent, / vellicent, vocent, molesti sint; occentent ostium:/ impleantur elegeorum meae fores carbonibus. ${ }^{457}$

Übersetzung: Weil ihre Erscheinung einen Skandal erzeugen würde, wenn sie der Mutter des Hauses folgt; wenn sie durch die Straßen ginge, würden alle sie anschauen, beobachten, ihr zunicken, oder -zwinkern, zischeln, sie zwicken,

455 Damit ist an dieser Stelle jedoch nicht das Phänomen der „Schriftbildlichkeit“, wie es von Sybille Krämer beschrieben wird (Krämer 2003; Krämer 2006), gemeint, sondern tatsächlich optische, ikonographisch zu beschreibende Eigenschaften von Buchstaben und Texten.

456 Vgl. auch: Solin 1979, 279; Franklin 1991, 84 Anm. 19; Zangemeister in CIL IV Hauptband S. V; Garrucci 1856, 5-6. Die zeitlichen Einordnungen der im Folgenden genannten Textstellen richten sich nach: Landfester 2007. Außer den hier zitierten Quellen finden sich einige weitere Textstellen, die sich auf die Anbringung von Graffiti im Rahmen öffentlicher aber anonymer Meinungsbekundungen im stadtrömischen Kontext beziehen, in Zadorojnyi 2011, 114-129.

457 Zitiert nach: T. Macci Plauti Comoediae recognovit brevique adnotatione critica instruxit W. M. Lindsay I (Oxford 1904). 
rufen, belästigen; vor der Tür Ständchen singen: Mein Hauseingang würde sich mit den Kohlezeichen der Elegien füllen.

Erzählzusammenhang: Demipho, Charinus’ Vater, äußert die Befürchtung, dass die Leute sich über seine Familie lustig machen und seine Tür bekritzeln könnten, wenn seine Frau mit der Hetäre Pasicompsa gesehen wird.

(2) Plaut. Rud.1294-1296 (201/200 v. Chr): cubitum hercle longis litteris signabo iam usquequaque, / si quis perdiderit vidulum cum auro et argento multo, / ad Gripum veniat. $^{458}$

Übersetzung: Beim Hercules, ich werde es gleich in ellenlangen Buchstaben überall anschreiben: Wenn jemand einen Koffer mit viel Gold und Silber verloren hat, soll er sich an Gripus wenden.

Erzählzusammenhang: Der Fischer Gripus hat einen Koffer mit Schmuck gefunden und sagt, er wolle überall Inschriften anbringen, damit derjenige, dem der Koffer gehört, sich an ihn wenden könne. ${ }^{459}$

(3) Cic. Verr. 2,3,77 (70 v. Chr.): de qua muliere versus plurimi supra tribunal et supra praetoris caput scribebantur. ${ }^{460}$

Übersetzung: Über diese Frau wurden viele Verse oberhalb des Tribunals und des Hauptes des Prätoren geschrieben.

Erzählzusammenhang: Pipa, die Frau des Syracusaners Aeschrion hatte anscheinend ein Verhältnis mit Verres. Dies führte dazu, dass am Amtssitz des Verres Graffiti über sie angebracht wurden.

(4) Prop. 3,23,23-24 (kurz nach 23 v. Chr.): i, puer, et citus haec aliqua propone columna, / et dominum Esquiliis scribe habitare tuum! ${ }^{461}$

Übersetzung: Lauf, Junge, und schreibe das schnell an irgendeine Säule! Und schreib, dass dein Herr auf dem Esquilin wohnt!

Erzählzusammenhang: Properz hat seine Schreibtafel, die bis dahin zum Austausch von Liebesbotschaften gedient hatte, verloren. Nun fordert er einen Sklaven auf, einen Graffito zu schreiben, um den Verlust zu melden, sodass ein eventueller Finder die Tafel zurückbringen könnte. Als Anbringungsort wird eine Säule empfohlen. Die Angabe des Wohnortes kann als Hinweis darauf verstanden werden, dass derartige Gesuche tatsächlich gelesen und ernstgenommen wurden. ${ }^{462}$

458 Zitiert nach: T. Macci Plauti Comoediae recognovit brevique adnotatione critica instruxit W. M. Lindsay II (Oxford 1905).

459 Vgl. Marx 1928, 220.

460 Zitiert nach: M Tulli Ciceronis scripta quae mansuerunt omnia volumen V. In Q. Caecilium divinatio. In C. Verrem actio I et II recognovit A. Klotz (Leipzig 1923).

461 Zitiert nach: Sex. Propertii elegiarum libri IV edidit Rudolf Hanslik (Leipzig 1979).

462 Paolo Fedeli deutet den Schluss als eine Aufforderung an den Sklaven, genau das eben beendete Gedicht als Suchmeldung an eine Säule zu schreiben: Fedeli 1985, 670. 
(5) Plin. epist. 8,8,7 (98-111 n. Chr.): nam studebis quoque: leges multa multorum omnibus columnis, omnibus parietibus inscripta, quibus fons ille deusque celebratur. plura laudabis, non nulla ridebis; quamquam tu vero, quae tua humanitas, nulla ridebis. ${ }^{463}$

Übersetzung: Denn Du wirst dort auch Untersuchungen anstellen können: Du wirst viele Aufschriften von ebenso vielen Schreibern an allen Säulen und allen Wänden lesen, in denen jene Quelle und der Gott gepriesen werden. Die meisten wirst Du loben, einige belächeln; ach nein, bei Deiner Gutmütigkeit wirst $\mathrm{Du}$ keine belächeln.

Erzählzusammenhang: Plinius beschreibt die Quelle des Clitumnus mit dem zugehörigen Heiligtum in einem Brief an Romanus. Die Graffiti werden hier explizit als zum Heiligtum gehörig beschrieben, und ihre Erwähnung bildet nicht nur den Schluss des Briefes, sondern auch die Gelegenheit, den wohlwollenden Charakter des Briefempfängers Romanus zu loben.

(6) Petron.71,11-12 (neronisch): horologium in medio, ut quisquis horas inspiciet, velit nolit, nomen meum legat. Inscriptio quoque vide diligenter si haec satis idonea tibi videtur: ,C. Pompeius Trimalchio Maecenatianus hic requiescit. Huic seviratus absenti decretus est. Cum posset in omnibus decuriis Romae esse, tamen noluit. Pius, fortis, fidelis, ex parvo crevit, sestertium reliquit trecenties, nec unquam philosophum audivit. Vale: et $\mathrm{tu}^{6}{ }^{\mathrm{4}}{ }^{44}$

Übersetzung: Eine Sonnenuhr in der Mitte, damit jeder, der nach der Uhr sieht, ob er will oder nicht, meinen Namen liest. Überlege auch gründlich, ob dir diese Inschrift passend genug erscheint: ,Hier ruht C. Pompeius Trimalchio Maecenatianus. Er wurde in Abwesenheit in den Sechserrat aufgenommen. Er hätte zwar in jedem Kollegium Roms Mitglied sein können, wollte aber nicht. Fromm, tüchtig, treu. Er ist aus kleinen Verhältnissen aufgestiegen. Er hat dreißig Millionen Sesterzen hinterlassen und hat niemals einen Philosophen gehört. Lebe wohl: auch Du!

Erzählzusammenhang: Trimalchio beschreibt während seiner cena, wie er sich sein Grabmal vorstellt. Als weitere bzw. wichtigste Inschrift sieht er allerdings die Formel: hoc monumentum heredem non sequatur (71.8) an.

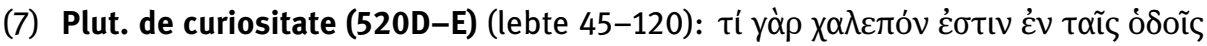

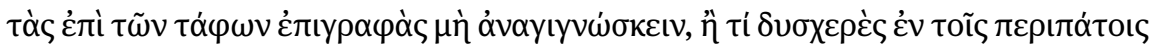

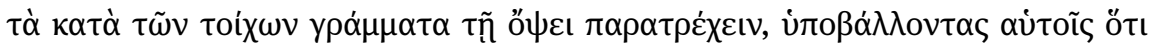

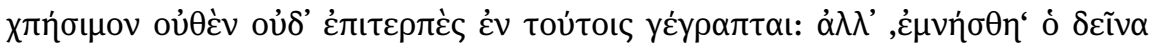

463 Zitiert nach: C. Plini Secundi epistularum libri novem, epistularum as Traianum liber, panegyricus recensuit Mauritius Schuster editionem tertiam curavit Rudolphus Hanslik (Leipzig 1992).

464 Zitiert nach: Petronii Arbitri Satyricon reliquiae quartum edidit Konrad Mueller (Leipzig 1995). 


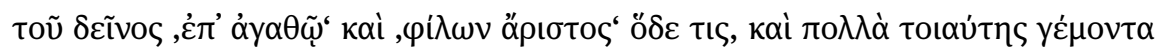

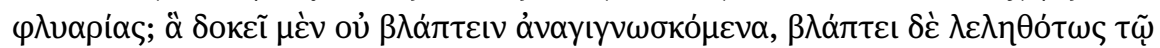

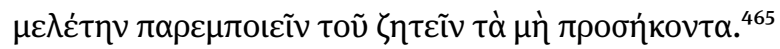

Übersetzung: Was ist denn so schwierig daran, unterwegs das auf die Gräber Geschriebene nicht zu lesen? Oder was so hart daran, bei Spaziergängen an den Wandinschriften vorüberzugehen? Wir müssen uns selbst nur vor Augen führen, dass dort nichts Nützliches oder Erfreuliches geschrieben steht: sondern ,irgendwer denkt an irgendwen', ,alles Gute', irgendeiner ist der ,beste Freund' und viele weitere Albernheiten. Es scheint vielleicht, als würde das Gelesene nicht schaden, aber es schadet doch unmerklich, indem es die Aufmerksamkeit auf Begebenheiten lenkt, die gar nicht präsent sind.

Erzählzusammenhang: Plutarch empfiehlt es als eine Übung, die dem übermäßigen Interesse für unwichtige Dinge entgegenwirken soll, im Vorbeigehen weder die Grabinschriften noch etwaige Graffiti zu lesen. Dabei hat er auch den Inhalt der Graffiti im Blick, nämlich persönliche Angelegenheiten, wie zum Beispiel Grüße, die für den Passanten keine Bedeutung haben sollten.

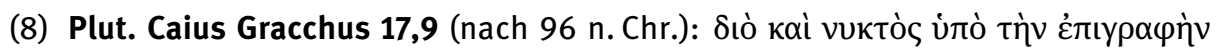

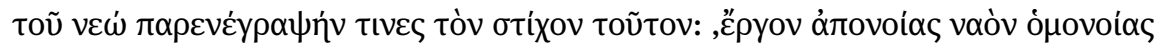

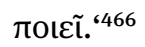

Übersetzung: Daraufhin fügten Unbekannte eines Nachts unterhalb der Inschrift des Tempels die folgende Zeile hinzu: ,Das Werk der Zwietracht baut der Eintracht einen Tempel.

Erzählzusammenhang: Graffiti werden in dieser Textstelle als Mittel des Widerstandes zitiert, wobei ein einzelner spezifischer Fall benannt wird. Ein anonymer Schreiber fügte den Graffito zur Inschrift am Concordia-Tempel hinzu, den Opimius erbaut/restauriert hatte, nachdem er für den Tod des Caius Gracchus und zahlreicher seiner Anhänger verantwortlich geworden war.

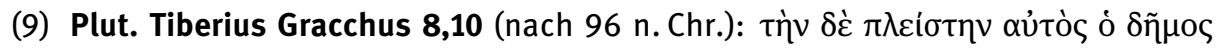

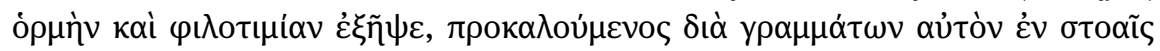

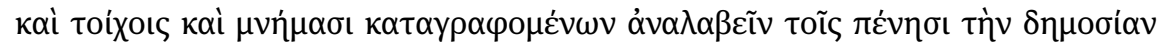
$\chi \omega \dot{\rho} \alpha v^{467}$

Übersetzung: Am meisten jedoch fachte das Volk selbst die Energie und den Ehrgeiz [des Tiberius] an, indem er durch Inschriften, die in Säulenhallen, Wände

465 Zitiert nach: Plutarchi Moralia vol.III recensuerunt et emendaverunt W. R. Paton, M Pohlenz, W. Sieveking (Leipzig 1972).

466 Zitiert nach: Plutarchi vitae parallelae recognoverunt Cl. Lindskog et K. Ziegler vol. III Fasc. 1 iterum recensuit K. Ziegler (Leipzig 1971) (Dort fortlaufende Zählung zwischen Tiberius und Caius: = 38.9).

467 Zitiert nach: Plutarchi vitae parallelae recognoverunt Cl. Lindskog et K. Ziegler vol. III Fasc. 1 iterum recensuit K. Ziegler (Leipzig 1971). 
und Monumente eingeritzt wurden, dazu aufgerufen wurde, das Gemeindeland für die Armen wiederzuerlangen.

Erzählzusammenhang: Tiberius Gracchus beginnt, sich für die Agrarreform zu engagieren. Er wird dadurch angestachelt, dass viele Leute Graffiti in Säulenhallen, an Wände und an Monumente schrieben.

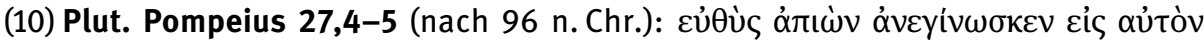

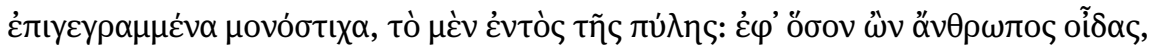

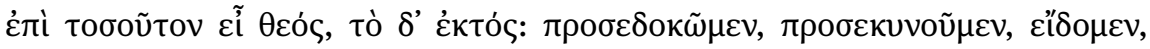
$\pi р о \pi \varepsilon \dot{\mu} \pi о \mu \varepsilon$. $^{468}$

Übersetzung: Als er gerade aufbrach, erkannte er zwei an ihn selbst gerichtete, eingeritzte Verse. Der an der Innenseite des Tores lautete: ,So sehr Du weißt, dass Du ein Mensch bist, so sehr bist Du ein Gott' und der an der Außenseite: ,Wir erwarteten Dich, wir grüßten Dich, wir haben Dich gesehen, wir geleiten Dich.' Erzählzusammenhang: Pompeius legt während seines Kampfes gegen Piraten einen Zwischenstopp in Athen ein. Als er die Stadt wieder verlässt, sieht und liest er zwei Graffiti.

(11) Suet. Nero 45,2 (120 n. Chr.): ascriptum et columnis, etiam Gallos eum cantando excitasse. 469

Übersetzung: Und auf Säulen wurde geschrieben, er habe mit seinem Gesang sogar die Hähne aufgeweckt.

Erzählzusammenhang: Das Schreiben von Graffiti ist Teil einer Reihe von Aktionen im öffentlichen Raum, die die Leute unternahmen, nachdem Nero durch besonders anmaßendes Verhalten offenen Hass auf sich gezogen hatte.

(12) Mart. 12,61,7-11 (86-102 n. Chr.): Quaeras censeo, si legi laboras, / nigri fornicis ebrium poetam, / qui carbone rudi putrique creta / scribit carmina, quae legunt cacantes. / Frons haec stigmate non meo notanda est. ${ }^{470}$

Übersetzung: Wenn Du von Dir lesen willst, so rate ich Dir einen betrunkenen Dichter der schwarzen Gewölbe zu fragen, der mit einem Kohlestückchen oder einer mürben Kreide Verse schreibt, die die kackenden lesen. Diese Stirn ist es nicht wert, dass sie durch ein Brandmal von mir gezeichnet wird.

Erzählzusammenhang: Martial schreibt ein Gedicht über Ligurra, in dem er klarstellt, dass dieser es nicht wert sei, mit einem Schmähgedicht bedacht zu werden,

468 Zitiert nach: Plutarchi vitae parallelae recognoverunt Cl. Lindskog et K. Ziegler vol. III Fasc. 2 iterum recensuit K. Ziegler (Leipzig 1973).

469 Zitiert nach: C. Suetonius Tranquillus opera vol.I. De vita Caesarum libri VIII recensuit Maximilian Ihm (Leipzig 1933).

470 Zitiert nach $M$. Val. Martialis epigrammata recognovit brevique adnotatione critica instruxit W. M. Lindsay (Oxford 1929). 
sondern allenfalls in Spottgraffiti in der latrina vorkommen werde. Dabei wird nicht nur der Inhalt und der Anbringungsort der Graffiti negativ charakterisiert, sondern auch der mögliche Autor wird als ebrius attribuiert.

(13) Lukian. Dial. Mer./Het. dial. 4,2-3 (lebte zwischen 115 (?) und 190):

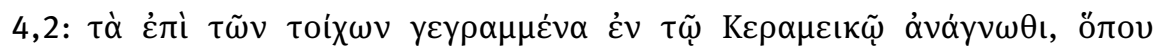

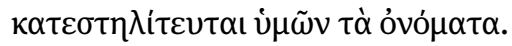

Übersetzung: Lies, was auf die Mauern im Kerameikos geschrieben ist, wo eure Namen eingeschrieben sind.

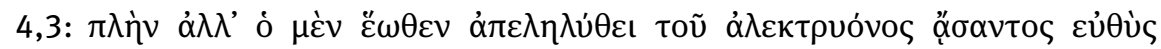

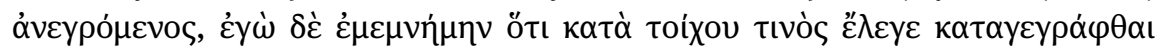

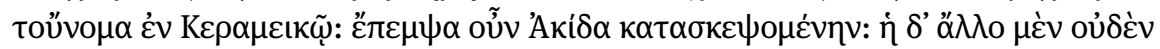

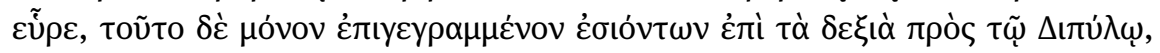

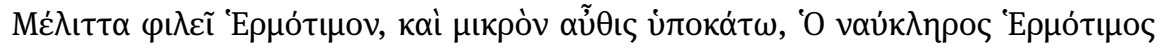
$\varphi \iota \lambda \varepsilon \tilde{~} M \varepsilon \dot{\lambda} \iota \tau \tau \alpha v^{471}$

Übersetzung: Aber früh am Morgen war er schon aufgebrochen, aufgeweckt durch das Krähen des Hahnes. Ich aber erinnerte mich, dass er gesagt hatte, dass auf irgendeine Mauer im Kerameikos die Namen geschrieben seien. Also schickte ich Akis los, um nachzusehen. Sie fand aber nichts außer diesem dort geschrieben, zur Rechten, wenn man durch das Dipylontor hineingeht: ,Melitta liebt Hermotimos' und wiederum kleiner darunter: ,Der Schiffseigner Hermotimos liebt Melitta'.

Erzählzusammenhang: Die Hetäre Melitta erklärt ihrer Freundin Bacchis, dass ihr Freund Charinus sich von ihr getrennt habe, nachdem er Graffiti gefunden hatte, in denen Melitta einem anderen ihre Liebe erklärt. Charinus scheint die Graffiti zufällig entdeckt zu haben. Akis, die von Melitta geschickt wird, um Charinus' Aussage zu verifizieren, muss jedoch nach den Graffiti suchen.

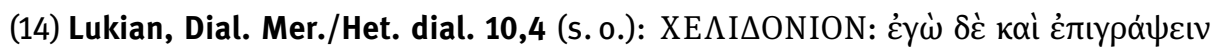

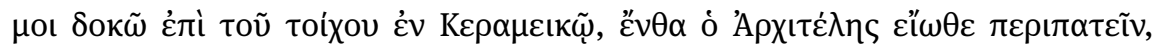

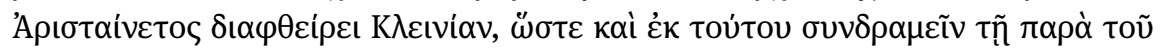

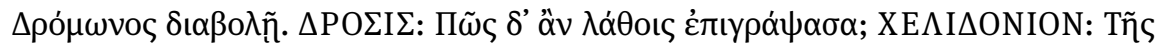

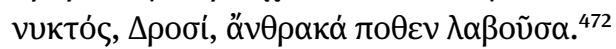

Übersetzung: CHELIDONION: Ich habe vor, auf eine Wand im Kerameikos zu schreiben, wo Architeles für gewöhnlich spazieren geht: ,Aristainetos verführt Kleinias.‘, sodass wir darin in der Verunglimpfung des Dromo übereinstimmen.

471 Zitiert nach: Luciani opera recognovit brevique adnotatione critica instruxit M. D. Macleod 4 (Oxford 1987).

472 Zitiert nach: Luciani opera recognovit brevique adnotatione critica instruxit M. D. Macleod 4 (Oxford 1987). 
DROSIS: Wie kannst Du das schreiben, ohne bemerkt zu werden? CHELIDONION: Des Nachts, Drosis, mit Kohle, die ich irgendwo hernehmen muss.

Erzählzusammenhang: Die Hetäre Drosis vermisst ihren Liebhaber Kleinias, der nicht mehr zu ihr kommt, weil sein neuer Lehrer Aristainetos ihm dies verboten hat. Drosis und ihre Freundin Chelidonion vermuten, dass Aristainetos Kleinias selbst verführen will. Um ihn zu denunzieren, plant Chelidonion, eine Anschuldigung an eine Wand im Kerameikos zu schreiben.

\subsubsection{Auswertung der litararischen Quellen}

Die aufgeführten Zitate entstammen ganz unterschiedlichen Erzählzusammenhängen und „Textsorten“, in denen die Graffiti bzw. Inschriften jeweils unterschiedliche Rollen spielen. Diese sollen im Folgenden anhand von Einzelaspekten beschrieben werden. Je nach Textgattung ist die Erwähnung der Inschriften mit unterschiedlichen erzählerischen Funktionen zu verknüpfen. Die in Klammern angegebenen Nummern verweisen auf die jeweilige Quelle.

\subsubsection{An der Anfertigung der Inschriften beteiligte Personen}

In den Textstellen werden Personen, die unterschiedlichen sozialen Schichten angehörten, benannt, die an der Anbringung der Inschriften beteiligt waren. In den meisten Fällen handelt es sich allerdings um größere Gruppen und anonyme Schreiber. Bei Plautus (1) sind es omnes, Cicero (3) gibt die Schreiber indirekt durch das Verb scribebantur im Passiv an. Bei Plutarch (10) wird kein konkreter Schreiber benannt. In (8) und bei Sueton (11) werden die Schreiber gezielt anonymisiert, da es sich um subversive Akte handelt. ${ }^{473}$ Eine zwar große aber dennoch eindeutig bezeichnete Gruppe stellt der bei Plutarch (9) benannte $\delta \tilde{\eta} \mu o \varsigma$ dar, der in einer konkreten Situation kollektiv tätig wird. Bei Plutarch (7) werden zwar keine Schreiber genannt, da in dem Textabschnitt aber dazu aufgefordert wird, kein Interesse an den belanglosen Angelegenheiten anderer $\mathrm{zu}$ verfolgen, findet hier eine deutliche Abgrenzung von den Schreibern als den Anderen statt. Die multi, welche in Plinius (5) auf die Wände geschrieben haben, können zwar nicht benannt, aber doch anhand des Anbringungsortes und der Inhalte als Besucher des Heiligtums eingeordnet werden.

Einzeln als Schreiber bezeichnet werden Sklaven in (2) und (4), wobei nur der Sklave des Properz im Auftrag seines Herrn handelt, der ihm genaue Anweisungen erteilt. Eine Hetäre ist die Schreiberin bei Lukian (14). ${ }^{474}$ Dies trifft möglicherweise auch auf Lukian (13) zu, hier ist aber gerade die falsche Zuordnung der Autorenschaft

473 Vgl. Zadorojnyi 2011, 125.

474 Besonders die Beteiligung von Frauen betont Milnor: Milnor 2014, 201-202. Vgl. Zadorojnyi 2011, 127; Harris 1989, 260-262. 
ein Hauptmotiv des Dialogs. Bei Petron (6) wird die Grabinschrift des Trimalchio thematisiert und hier zeigt sich, dass bei den Steininschriften mit einer Trennung der Person, die den Text formulierte, von der, die die Inschrift am Monument anbrachte zu rechnen ist. ${ }^{475}$

In den Augen der antiken Autoren war das Schreiben von Graffiti demnach nicht auf bestimmte Personengruppen beschränkt. Insbesondere scheinen Gender und der Status als Sklave, Freigeborener oder Freigelassener keine ausschlaggebenden Kriterien darzustellen. Vielmehr erwecken summarische Zuordnungen wie omnes, multi oder $\delta \tilde{\eta} \mu o \varsigma$ den Eindruck, dass die Autoren davon ausgingen, dass theoretisch alle an dieser Praxis teilnehmen konnten. ${ }^{476}$

\subsubsection{Anbringungsorte}

Mehrfach wird in den Quellen ein enger Bezug zu einzelnen Bildwerken, zu bestimmten Orten oder Bereichen der Stadt oder auch zu spezifischen Gebäuden betont, mit denen die Inschriften verbunden wurden. ${ }^{477}$ Die genannten Orte liegen fast alle im öffentlichen Raum oder an der Grenze dazu. Lediglich der fornix bzw. die latrina (12) könnten sich auch in einem privaten Gebäude befunden haben. Als Übergriff in den persönlichen Raum wird in (1) offenbar auch das Beschreiben des Hauseingangs empfunden, auch wenn dieser frei zugänglich war. Bei den Säulen und Wänden des Clitumnus-Heiligtums ist es denkbar, dass die Beschriftung explizit geduldet wurde oder sogar Teil der rituellen Praxis war, da es dort theoretisch leicht gewesen wäre, solche Aktivitäten zu unterbinden. Insgesamt werden meist Säulen oder Wände und Denkmäler als Beschreibflächen benannt ((4), (7), (9), (10)). Nur in (2) wird pauschal usquequaque geschrieben. Präzisere Benennungen finden sich für die in Athen situierten Texte, die alle im Kerameikos und zwar am Dipylontor oder in dessen Nähe angebracht werden/waren: (10), (13) und (14). Ebenfalls konkret angegeben wird auch eine Stelle unterhalb der Inschrift des Concordia-Tempels in Rom (8) sowie mit Bezug auf die gegen die Frau des Aeschrion gerichteten Graffiti supra tribunal et supra praetoris caput.

Fraglos steht die Art, wie der Anbringungsort bezeichnet wird, in engem Zusammenhang mit der erzählerischen Absicht der jeweiligen Autoren: Die Absicht, überall zu verkünden, dass ein Koffer voll Wertsachen gefunden wurde, steht im Kontrast

475 Vgl. dazu Solin 1979, 277. Auch Langner betont, dass sich immer wieder Hinweise auf eine planvolle Vorgehensweise finden: Langner 2001, 25.

476 Auch eine Beschränkung auf Jugendliche, wie Kruschwitz und Halla-aho sie annehmen, wird weder durch die Quellen noch durch die Graffiti selbst gestützt: „If one believes in certain human constants throughout the ages (like, we all walk upright, from a certain age onwards') it seems justified to say that even in antiquity it will have been the young rather than the old who wrote obscenities and witticisms on the wall“: Kruschwitz/Halla-Aho 2007; auch: Kruschwitz 2010.

477 Vgl. dazu auch die von Zadorojnyi zitierten Quellen mit einem spezifisch stadtrömischen Bezug: Zadorojnyi 2011, 121-122. 125. 
zu der eigentlich erwünschten Geheimhaltung. Die Bezeichnung aliqua columna bei Properz erklärt sich aus der Eile, in der der Sklave losgeschickt wird, um die verlorene Tafel wiederzuerlangen. Und als Historiker muss Plutarch ebenso wie Cicero in seiner Invektive Details so genau wie möglich benennen können, um glaubwürdig zu sein. Nicht nur im Vergleich mit den Befunden in Pompeji und Herculaneum, sondern auch bei der Synopse der vierzehn Textstellen erweisen sich jedoch besonders Wände, Säulen, Tür- und Torbereiche sowie latrinae als charakteristische Anbringungsorte.

\subsubsection{Materialien und Gestaltung}

Das Material und die Gestaltung der Wandinschriften werden meist nicht thematisiert. Explizit wird in (1), (12) und (14) Kohle genannt, in (12) darüber hinaus auch noch Kreide. Ebenso spärlich sind die Angaben zur Gestaltung. Lediglich Gripus kündigt an, er werde die Bekanntmachung mit cubitum longis litteris schreiben (2). Dies ist natürlich eine Übertreibung. Ähnliche Buchstabenhöhen sind bei Graffiti zwar in Einzelfällen belegt, fördern jedoch nicht die Lesbarkeit, da Strichdicke und Länge der Hasten in einem ungünstigen Verhältnis zueinander stehen. ${ }^{478}$

\subsubsection{Themen, Inhalte und Anlässe für die Anbringung von Inschriften}

Die Inhalte werden in den meisten Quellen benannt. Teilweise werden sie wörtlich zitiert: (2), (6), (7), (10), (11), (13) und (14); in den anderen Fällen wird der Inhalt paraphrasiert oder mit einem Schlagwort eingeordnet. In (5) erfahren wir lediglich, dass Quelle und Gott gepriesen wurden.

Mehrfach werden Graffiti genutzt, um Spott und üble Nachrede über einzelne Personen oder politische Kommentare im öffentlichen Raum anzubringen: (3), (8), (9), (11), (12) und (14). Diese Meinungsbekundungen dienen dabei teilweise dazu, entweder den Angesprochenen, wie Tiberius Gracchus, oder auch Dritte, wie in Lukians Dialog von Chelidonion intendiert, zu weiteren Handlungen $\mathrm{zu}$ animieren. Mit den Graffiti sollte also direkt Einfluss auf das öffentliche Leben und Personen von öffentlichem Interesse genommen werden. Besonders bei Cicero und Sueton aber auch im Zusammenhang mit dem Graffito am Concordia-Tempel steht dagegen die Möglichkeit im Vordergrund, sich trotz einer als erdrückend oder bedrohlich empfundenen Herrschaftsform, im Schatten der Masse und der Anonymität zu Wort zu melden und das Verhalten des Herrschers zu kommentieren. Der Leserschaft kommt dabei auch eine Kontrollfunktion zu: Die „Leute“ waren nicht nur Rezipienten der Texte, sondern auch Zeugen der Reaktionen direktangesprochener Personen.

Liebesschwüre (13) und Epigramme bzw. Elegien (1) werden ebenfalls als Inhalte benannt, wobei die Elegien von Plautus in einen direkten Zusammenhang mit den

478 Vgl. CIL IV 10564 in den fauces der Casa Sannitica in Herculaneum. Die im CIL angegebene Höhe von $19 \mathrm{~cm}$ trifft nicht zu. Richtig sind: $28 \mathrm{~cm}$. 
mündlich (occentent) dargebrachten paraklausithyra gesetzt werden. Diese Inhalte sind in besonderem Maße mit einem bestimmten Ort verknüpft: einerseits mit der Tür der Person, an die ein Epigramm gerichtet werden sollte, und andererseits hoffte der Schreiber wie bei Lukian, dass die Geliebte ihrerseits eine Antwort hinzufügen würde.

Besonders solche Inhalte, die die Beziehungen der beteiligten Personen thematisieren, oder die in den historischen Texten das Handeln einzelner in einen größeren Zusammenhang des zeitgenössischen Diskurses stellen, sind wie die genannten Orte eng mit den erzählerischen Vorgehensweise verknüpft. So kann der Plan, einen Graffito anzubringen, wie von Lukian im Dialog zwischen Chelidonion und Drosis, gezielt als Prolepse eingesetzt werden, um den möglichen Fortgang der Begebenheit noch im Rahmen des Dialoges aufzeigen zu können. Die Anbringung der Graffiti bei Plautus (2) und Properz (4), die auf verlorenes oder gefundenes Gut aufmerksam machen, deuten zugleich die Möglichkeit an, dass dieses wieder erhalten werden könnte. Die Inhalte sind daher im Zusammenhang mit der übergeordneten Handlung zu sehen. Bei Plutarch (7) steht hingegen gerade die Absicht im Vordergrund, ganz gewöhnliche Wandinschriften zu benennen, die überall und dezidiert ohne Bezug zu dem gedachten Spaziergänger angebracht waren. Die von ihm genannten Beispiele beziehen sich auf persönliche Angelegenheiten und freundschaftliche Verhältnisse zwischen Individuen. Auch hier finden sich direkte Anreden an einzelne Personen.

\subsubsection{Verhaltensweise der Schreiber}

Die Inschriften werden in den Texten kaum gattungsspezifisch klassifiziert, sondern

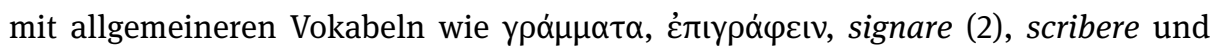
inscripta benannt. Genauere Angaben oder Hinweise zum Vorgehen der Schreiber finden sich bei Cicero (3), Properz (4), Plutarch (8) und Lukian (14). Cicero verwendet das Imperfekt scribebantur, was auf eine lange Dauer oder Wiederholung der Praxis hindeutet. Properz weist seinen Sklaven an, citus - schnell - zu schreiben. Bei Plutarch und Lukian wird explizit erwähnt, dass nachts geschrieben wurde. Allerdings spielt in beiden Fällen das Ziel, den Graffito heimlich anzubringen, eine vordringliche Rolle, sodass das Schreiben bei Nacht nicht die Regel, sondern eher die Ausnahme gewesen zu sein scheint.

\subsubsection{Rezeption und Beurteilung der Inschriften}

Gerade im Zusammenhang mit Graffiti wird oft angenommen, dass das Schreiben solcher Texte als deviant angesehen wurde. ${ }^{479}$ In den hier zitierten Texten werden Graffiti unterschiedlich bewertet, was sich sowohl auf ihren Inhalt als auch auf ihr Erscheinungsbild beziehen kann. Auch innerhalb eines Textes werden unterschiedliche

\footnotetext{
479 Vgl. Bagnall 2011, 9. Roger Bagnall geht davon aus, dass Graffiti als „defacement“ wahrgenommen wurden. Auch: Voegtle 2012, 107. Dagegen Benefiel 2011, 20. 38-39.
} 
Beurteilungen formuliert. Bei Plautus (1) stehen die Wirkung und das Material Kohle im Vordergrund, welches durch die Menge an Elegien besonders auffällig wird. Plutarch (7) beurteilt die Inhalte kritisch mit dem Begriff $\varphi \lambda v \alpha \rho i \alpha \varsigma$. Die Graffiti bei Properz (4) und Plautus (2) hingegen werden als adäquate Hilfsmittel genutzt, um sich an größere Gruppen zu wenden. Das Urteil der handelnden Personen und der Erzähler über die Graffiti ist also heterogen. Je nach Situation werden sie als selbstverständlich, als geeignetes Mittel zu einem spezifischen Zweck oder als Störfaktoren aufgefasst.

Legere und $\dot{\alpha} v \alpha y \iota v v \omega \dot{\omega} \sigma \kappa \varepsilon \imath v$ sind die meist gebrauchten Verben, die sich auf die Wahrnehmung der Texte beziehen. Aber Graffiti können offenbar auch wahrgenommen werden, ohne dass sie in ihrem Wortlaut gelesen wurden. Sie werden zunächst zufällig entdeckt oder gesucht (13) und in der Folge mit anderen verglichen, gelobt oder belächelt (5), beantwortet (13) und bewertet. Einige dieser möglichen Reaktionen werden antizipiert, wenn zum Beispiel Properz damit rechnet, dass jemand seinen Aufruf sehen und daraufhin $\mathrm{zu}$ ihm kommen werde, oder wenn Chelidonion davon ausgeht, dass sie Aristainetos durch einen bloßstellenden Graffito schädigen könne. Die Wandinschriften werden in den Textstellen auch als Bestandteil von Gesprächsthemen eingebracht und werden inhaltlich so ernst genommen, dass sie Beziehungen zwischen Menschen beschädigen können (13), (14). Besonders Plutarch (7) thematisiert die Möglichkeit, die Präsenz eines Graffito wahrzunehmen, ihn jedoch nicht

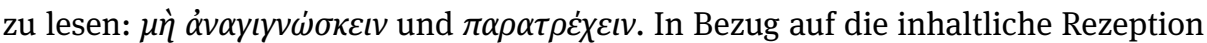
warnt Plutarch hier davor, sich zu intensiv mit den Graffiti auseinanderzusetzen, da sie Sachverhalte thematisieren, die nicht für jeden von Belang seien. Implizit wird durch die Schilderung des Graffito am Concordia-Tempel ausgesagt, dass solche Inschriften, die durch Bezüge zu einflussreichen Personen, Gebäuden und historischen Situationen in einen bedeutsamen Kontext eingebettet waren, auch das Potential hatten, Eingang in eine längerfristige Überlieferung zu finden. ${ }^{480}$

Der Leserkreis wird meist nicht spezifiziert. Lediglich bei Plutarch (7) und (10), Martial (12) und Lukian (13) werden Leser explizit erwähnt. Plutarch wendet sich zum einen an den impliziten Leser seiner ethischen Belehrungen (7). Zum anderen nennt er Pompeius (10), der - ohne dass der moderne Leser erfährt, woran - erkennt, dass sich die zitierten Graffiti auf ihn beziehen. Lukian beschreibt Melitta und deren Dienerin Akis als potentielle bzw. tatsächliche Leserinnen. Martial nennt mit den cacantes eine nicht personell, aber doch situativ einzugrenzende Gruppe.

\subsubsection{Erzählerische Funktion der Graffiti}

Fragt man nach der erzählerischen Funktion der Graffiti innerhalb der Texte, scheint dies zunächst für eine kulturhistorische Auswertung realer Graffiti wenig gewinnbringend. Entweder handelt es sich ohnehin um fiktionale Texte oder die Existenz der

480 Solin erklärt dies unter anderem dadurch, dass sich Graffiti mit großer Aktualität auf einzelne Ereignisse und Personen von öffentlichem Interesse beziehen konnten: Solin 1979, 279. 
konkret benannten Inschriften ist im Befund nicht nachzuweisen. Dennoch sind sie mehr als bloß der Ausschmückung dienender Zierrat. Vielmehr zeigt ihre Verortung innerhalb der Erzählungen, dass das Anbringen, Lesen oder Besprechen von Graffiti innerhalb der geschilderten Handlungszusammenhänge plausibel war. Anhand der erzählerischen Funktion können daher Beurteilungen gewonnen werden, die über die explizit formulierten, im vorangehenden Abschnitt besprochenen Äußerungen hinausgehen.

Sowohl in der Analepse als auch in der Prolepse, also in der Rückschau und in der Vorwegnahme von Zukünftigem auf der Erzählebene, erhalten die Graffiti Qualitäten, die ihnen eine über die geschilderte Handlung zeitlich hinausgehende Bedeutung verleihen. Durch sie wird ein Rahmen hergestellt, der zeitlich zwar nicht genau definiert ist, aber über die Haupthandlung hinausreicht. Als vor der Handlung existente Schriftzeugnisse bilden sie den Ausgangspunkt für das weitere Geschehen oder bieten den Handelnden Anlass zur Reflexion. In der Vorwegnahme von Rezeptionsvorgängen oder des Anbringens neuer Graffiti werden gegenwärtige Aktionen mit zukünftigen verknüpft, wobei die Planung oder Thematisierung der Graffiti das Bindeglied zwischen den verschiedenen Momenten bildet.

Auf der Handlungsebene wird durch Graffiti eine Kommunikation mit Abwesenden möglich, die dennoch als eine persönliche Art der Kommunikation empfunden wurde. Dies zeigt sich besonders dadurch, dass explizit über Reaktionen von Beteiligten und Unbeteiligten reflektiert wird bzw. dass die Graffiti als Reaktionen auf ein Geschehnis vorgestellt werden.

Im Verhältnis zu den in den Quellen wiedergegebenen Dialogen der handelnden Figuren sowie Äußerungen, die Individuen oder Personengruppen zugeschrieben werden, erhalten die Graffiti grundsätzlich andere Qualitäten. Zwar handelt es sich bei Ihnen im Allgemeinen um Konkretisierungen, Profilierungen und Materialisierungen sprachlicher Äußerungen. Aber in keinem Fall sind die Graffiti eine Verschriftlichung einer mündlichen Äußerung. Bei Plautus (1) wirkt sogar die schriftliche Äußerung auf die mündliche zurück: Gripus plant nicht eine mündliche Äußerung zu verschriftlichen, sondern er spricht eine vorformulierte, geplante schriftliche Äußerung mündlich aus. Ähnliches gilt für Properz (4). Auch bei Plautus (2) wird deutlich zwischen mündlichen und schriftlichen Äußerungen unterschieden: occentent und elegeorum carbonibus. Während in diesen Textstellen eine enge wechselseitige Beziehung zwischen mündlichen und schriftlichen Äußerungen besteht, spielt diese bei Plinius (5), Cicero (3) und Plutarch (7) keine Rolle. Hier wird nicht thematisiert, ob mit der Anbringung von Graffiti mündliche Äußerungen einhergingen oder ähnliche Inhalte auch ausgesprochen wurden. In keinem Fall werden die Graffiti als spontane Äußerungen dargestellt, denen eine ähnliche Impulsivität zuzusprechen wäre wie etwa einem mündlichen Ausruf oder der spontanen Reaktion in einem Gespräch. Vielmehr handelt es sich um kalkulierte und kalkulierbare Praktiken, die den handelnden Personen offenbar geläufig waren. Abgesehen von dieser grundsätzlichen Unterscheidung zwischen den beiden Formen sprachlicher Äußerungen, wird in den Quellen besonders 
das Potenzial von Graffiti betont, sprachliche Äußerungen mit ganz bestimmten Orten in Beziehung zu setzen, die jeweils für die Art der Rezeption konstitutiv sind.

\subsection{Inschriften in Pompeji und Herculaneum}

Auch wenn im Grunde jede Inschrift in einem metatextuellen Verhältnis zu sich und $\mathrm{zu}$ allen anderen Inschriften steht, da immer wieder inhaltliche und formale Muster rezipiert, reproduziert und mit ihrer Reproduktion als valide Konventionen dargestellt werden, ${ }^{481}$ gibt es einige Inschriften, die inhaltlich oder formal in besonderem Maße eine Distanz zu anderen Texten erzeugen und sich somit explizit als Aussagen über Geschriebenes präsentieren. Dazu gehört unter anderem ein vergleichsweise berühmter Graffito, der in Pompeji dreimal überliefert ist und in dem (andere) Wandinschriften als taedia bezeichnet und dadurch objektiviert werden. ${ }^{482}$ Aber wie Milnor bemerkt, zeigen viele Graffiti einen ,sense of themselves as artifacts“ und damit einen deutlichen metatextuellen Charakter. ${ }^{483}$ Die Bezüge, die in den Inschriften hergestellt werden, können auf unterschiedlichen Ebenen angesiedelt sein. Sie betreffen die bereits anhand der literarischen Quellen untersuchten Aspekte: den Herstellungsprozess, den Aufstellungsort, den Kontext oder in die Produktion involvierte Personen. Andere nehmen auf Inhalte anderer Inschriften oder des eigenen Textes Bezug.

\subsubsection{Der Altar des Marcus Nonius Balbus in Herculaneum}

Unter den Steininschriften weist besonders die Inschrift, die am Altar des Marcus Nonius Balbus in Herculaneum angebracht ist, metatextuellen Charakter auf. Der Text lautet:

[Qu]od M(arcus) Ofillius Celer IIvir iter(um) v(erba) f(ecit) pertinere at(!) municipi(i) / dignitatem meritis M(arci) Noni Balbi respondere d(e) e(a) r(e) i(ta) c(ensuerunt) / [cu]m M(arcus) Nonius Balbus quo hac vixerit parentis animum cum plurima liberalitat(e) / singulis universisque praistiterit placere decurionibus statuam equestrem ei poni quam / celeberrimo loco ex pecunia publica inscribique M(arco) Nonio M(arci) f(ilio) Men(enia) Balbo pr(aetori) proco(n)s(uli) patrono universus / ordo populi Herculanie(n)ssis(!) ob merita eius item eo loco quo cineres eius conlecti sunt aram / marmoream fieri et constitui inscribique publice M(arco) Nonio M(arci)f(ilio) Balbo exque eo loco parentalibu(s) / pompam duci ludisque gymnicis qui soliti errant fieri diem edici unum in honorem eius et cum in theatro / ludi fient sellam eius poni c(ensuerunt). ${ }^{484}$

481 Entsprechendes gilt auch, wenn Muster durchbrochen und Konventionen gerade nicht befolgt werden.

482 Siehe unten Kapitel 3.2.3.

483 Milnor 2014, 31.

$484 \operatorname{AE} 1947,53$ = AE 1976, 144. 
In dem Altar (Abb. F47) wurden Aschereste gefunden, die aller Wahrscheinlichkeit nach als Bestattung des Nonius Balbus selbst zu deuten sind. ${ }^{485}$ In der langen Inschrift, die an der südlichen, der Küste zugewandten Seite des Altars angebracht ist, ist von zwei Monumenten die Rede. ${ }^{486}$ Zum einen wird eine Reiterstatue genannt, die an einem locus celeberrimus aufgestellt werden sollte. Zum anderen wird als zweites Monument ein Altar erwähnt, in dem die Aschereste gesammelt sein sollten. Bei beiden erwähnten Monumenten ist zudem auch von je einer zugehörigen Inschrift die Rede (Abb. F48). ${ }^{487}$ Die Inschriften werden jeweils mit dem Terminus inscribi eingeführt, das sich auf die Anbringung an der Statue bzw. dem Altar bezieht. Im darauf folgenden Teil wird die geplante Inschrift im Wortlaut wiedergegeben. Die Inschrift (grün) für die Reiterstatue erstreckt sich über zwei Zeilen und nennt sowohl den Namen als auch die Ämter des Nonius Balbus und die Bürgerschaft Herculaneums als Stifterkollektiv. Die für den Altar angegebene Inschrift (orange) ist wesentlich kürzer. Sie besteht ausschließlich aus dem Namen des Verstorbenen. Ein Vergleich dieser beiden Inschriften und der Inschrift, in der sie vorkommen, also AE 1976, 144, zeigt, dass hier verschiedene Inhalte auf die beiden Ebenen verteilt wurden, indem man die Ämter des Nonius Balbus in der zitierten Inschrift, die an der Ehrenstatue anzubringen war, nannte und auf der ersten Ebene vor allem den Beschluss des Rates paraphrasierte. Die drei Texte stehen zueinander im Verhältnis einer verschachtelten mise en abyme, insofern als die beiden zitierten Inschriften zwar durch inscribi gerahmt und somit abgesetzt werden, die Inhalte der Ebenen jedoch ineinandergreifen. Sofern es nicht noch einen zweiten Altar für Nonius Balbus mit darin aufbewahrten Ascheresten gab, müsste es sich bei der zweiten zitierten Inschrift theoretisch um eben die lange Inschrift auf dem tatsächlich bekannten Altar handeln, die die beiden zitierten Inschriften enthält. Die Texte sind offensichtlich nicht identisch. Daher und mit Blick auf die Verteilung der Inhalte in mehrere Inschriften, kann nur geschlossen werden, dass mit den Angaben eher der Beschluss, Nonius Balbus mit außergewöhnlichen Monumenten zu ehren, wiedergegeben werden sollte, nicht jedoch die Festlegung auf einen bestimmten Wortlaut.

Die Inschrift lässt sich bezüglich der eingangs genannten Aspekte auswerten: beteiligte Akteure, Anbringungsort, Material und Gestaltung, Thema, Inhalt und Anlass sowie die Rezeption der Inschrift. Als beteiligte Akteure werden der Geehrte genannt, der die Ehrung durch seine herausragenden Leistungen verdient hatte. Den

485 Besonders zum Monument und der Bestattung: Pagano 1988a. Vgl. Maiuri 1958b, 148. Guidobaldi 2008, 63; Camodeca 1982, 125-126. Zur Inschrift und den darin genannten Monumenten vor allem: Guadagno 1978, 152 und Schumacher 1976; dazu auch: Zanker 1983, 260-264; Bergemann 1990, 125 Kat. E20.

486 Drei weitere Ehrungen, die in der Inschrift genannt werden, sind eine jährlich stattfindende Prozession an den parentalia, ein Ehrensitz im Theater und ein zusätzlicher Tag zu seinen Ehren bei öffentlichen Spielen: Vgl. dazu Schumacher 1976, 168-176, besonders: 173.

487 Vgl. Wallace-Hadrill 2011c, 130. 
Beschluss musste wie üblich der ordo decurionum des municipium fassen, wobei der ordo hier nur implizit mit der Formel $d(e) e(a) r(e) i(t a) c$ (ensuerunt) erwähnt wird. Als Antragsteller und somit Initiator wird Marcus Ofillius Celer benannt. ${ }^{488}$ Die Anbringungsorte der beiden Inschriften werden präzise angegeben. Zum einen eine Reiterstatue am celeberrimus locus, von dem wir nicht wissen, wo er sich befand und zum anderen der Altar selbst. ${ }^{489}$ Wie und wo genau die Inschriften anzubringen waren, wird nicht beschrieben. Das Material wird ebenfalls nicht bezeichnet. Thema, Inhalt und Anlass werden zwar ausführlich besprochen, allerdings kann es sich bei dem für den Altar zitierten Text nicht um den Wortlaut gehandelt haben, den man auf einem so wichtigen Monument hätte anbringen lassen. Dieser scheint mit der Veröffentlichung des kompletten Dekrets jedoch obsolet geworden zu sein. Die Wahrnehmung und mögliche Leser werden zwar nicht explizit benannt, aber dadurch, dass die eine Inschrift am celeberrimus locus stand und sich die andere am Ausgangspunkt des Festzuges der eigens neu organisierten parentali ${ }^{490}$ und zudem vor dem Eingang der Terme suburbane befand, war ein großer Rezipientenkreis impliziert.

Die Inschriften wurden ebenso wie die Statue bzw. der Altar explizit in die Beschreibung aufgenommen. Es wird deutlich, dass sie als integraler Bestandteil der Monumente und nicht als reine Beschriftungen der Ehrenstatuen gesehen wurden. Die inhaltlich starke Verkürzung vor allem der zweiten zitierten Inschrift ist zum Teil zwar der Komposition der gesamten Inschrift geschuldet. Sie gibt aber auch einen Hinweis darauf, welcher Teil der Inschrift der wichtigste war: die Zuschreibung an die geehrte und in diesem Fall auch bestattete Person. In dieser vermittelnden Rolle zeigt sich das performative Potential des eingeschriebenen Textes. Auch wenn diese Inschriften nur in Verbindung mit dem Gesamtmonument denkbar und letztlich verständlich sind, ist es dennoch gerechtfertigt, gerade weil sie hier wörtlich zitiert werden, nahe, von ihnen tatsächlich als „Ehreninschriften“ zu sprechen. ${ }^{491}$

\subsubsection{Schreibersignaturen}

Auch bei den Dipinti finden sich Texte, die als Metatexte bezeichnet werden können. Dazu zählen besonders die Signaturen mit der Formulierung „scripsit“, die sich bei zahlreichen programmata und edicta munerum finden und in engem Zusammenhang mit der Frage nach den beteiligten Akteuren stehen. ${ }^{492}$ Die meisten sind eindeutig auf

488 Dieser ist ansonsten kaum bekannt: AE 1959, 297 könnte eine Erwähnung seines Namens beinhalten, die Stelle ist jedoch korrupt und das cognomen nicht lesbar. Es handelt sich um eine juristische Urkunde aus Herculaneum. Ein Marcus Ofillius erscheint dort als erstgenannter und daher höchstrangiger Zeuge.

489 Zur Identifizierung der Statue: Schumacher 1976 und Zanker 1983.

490 exque eo loco parentalibu(s) / pompam duci.

491 Vgl. dagegen: Eck 1999, 67.

492 Vgl. dazu auch Kapitel 5.1.2. 
einen Dipinto zu beziehen, ${ }^{493}$ es kommen jedoch auch Doppelungen vor. ${ }^{494}$ Zuvorderst sind diese Anmerkungen natürlich als eine Art Signatur auf die jeweiligen Schreiber zu beziehen. Doch auch die Frage, was sie über die Dipinti aussagen, ist sinnvoll. Wie Sabbatini Tumolesi treffend zusammenfasst, sind sie zu verstehen als „far conoscere la paternità dell'opera eseguita““.495 Daran zeigt sich ein weiterer wichtiger Aspekt, nämlich, dass die Dipinti durch die Signaturen zu Werken wurden, die aufgrund der Fertigkeiten bestimmter Personen existierten und ein bestimmtes Aussehen hatten. Somit betont die Angabe des Malers den Artefaktcharakter dieser Wandinschriften. Auch bei Steininschriften kommen entsprechende Vermerke vor, aus Pompeji oder Herculaneum sind jedoch bisher keine Beispiele bekannt. ${ }^{496}$ Neben der Auszeichnung von Dipinti als Artefakte, nehmen die Schreibersignaturen auch Bezug auf deren ökonomische Bedeutung. Inschriften werden hier als Ware, die von professionellen und selbstbewussten Handwerkern angefertigt wurden, präsentiert. ${ }^{497}$

Während sich unter den Steininschriften und Dipinti somit eine überschaubare Gruppe an explizit metatextuellen Inschriften ausmachen lässt, ist der Befund in dieser Hinsicht bei den Graffiti zahlreicher und vielfältiger. Insbesondere sind hier drei Gruppen von Inschriften zu nennen: zum ersten, vergleichbar mit den scripsit-Angaben bei den Dipinti, Hinweise auf die Autoren, zum zweiten Texte, in denen explizit Bezug auf andere Inschriften genommen wird, und zum dritten - damit eng verbunden - normative Aussagen und insbesondere Verbote, an einer bestimmten Stelle Inschriften anzubringen. Letztere sind in Pompeji und Herculaneum nicht belegt, aber aus anderen Kontexten bekannt. ${ }^{498}$ Aus der zweiten Gruppe sollen drei sehr ähnliche Graffiti herausgegriffen und im folgenden Abschnitt behandelt werden. ${ }^{499}$ Unter den Zeugnissen, die der ersten Gruppe zuzuordnen sind, sind ein Cluster, das mit dem Namen Tiburtinus in Verbindung gebracht wird sowie einige Graffiti verschiedener Urheber und verschiedenen Inhaltes.

Die Graffiti CIL IV 4966-4973 wurden an der westlichen Außenwand des odeion gefunden. ${ }^{500}$ Die Putzschicht, in die sie eingeschrieben sind, war von einer weiteren Putzschicht überdeckt. Nach dem CIL waren sie Teil einer Wanddekoration 2. Stiles, an die die nördliche Begrenzung des ludus gladiatorius VIII 7, 16 anstößt. Nach der Auffindung wurden die Putzplatten von der Wand abgenommen und in das Museo Archeologico Nazionale di Napoli verbracht, wo sie sich auch heute noch befinden.

493 Fraglich bei CIL IV 7621. Vgl. dazu Ciprotti 1975, 274; Magaldi 1930, 52.

494 Z. B.: CIL IV 3884, Vgl. Cooley/Cooley 2013, 69.

495 Sabbatini Tumolesi 1980, 123. So auch: De Marchi 1916, 67.

496 Vgl. Chiavia 2002, 86; s. z. B.: AE 1971, 146; AE 1981, 131; AE 2000, 977.

497 Vgl. Chiavia 2002, 86-89.

498 Vgl. Solin 1979, 279. Z. B.: CIL VI 52 aus Rom. Vgl.: Kruschwitz 2010.

499 Siehe Kapitel 3.2.3.

500 CIL IV 4966=CLE 934; CIL IV 4967-4973=CLE 935. Bücheler rechnet jedoch die Signatur (in CIL IV 4966) dem zweiten Teil zu. Vgl. Morelli 2000, 104-107 Abb. 6-9. Milnor 2014, Taf. 2-5. 
Das Anstoßen der Wand und die Überlagerung durch eine neuere Putzschicht des ludus gladiatorius deuten darauf hin, dass die Graffiti im Jahr 79 n. Chr. bereits einige Jahre oder Jahrzehnte alt und mit Sicherheit nicht zu sehen waren. ${ }^{501}$ In den Putz sind für Graffiti vergleichsweise lange, aber teilweise fragmentierte Texte eingeritzt. Es ist nicht möglich, mit Sicherheit verschiedene Hände zu unterscheiden. Jedenfalls waren der oder die Schreiber versiert im Schreiben kursiver Buchstaben. ${ }^{502}$ Bei diesen klein und sorgfältig geschriebenen Zeilen handelt es sich um metrisch komponierte Verse, die anscheinend originäre Neuschöpfungen und keine Zitate anderer, bereits bekannter Dichtungen darstellen. ${ }^{503}$ Etwas beiseite und etwas größer geschrieben finden sich die Worte: Tiburtinus epoese, eine Formel, die vor allem aus der archaischen und klassischen griechischen Vasenmalerei und Skulptur bekannt ist. Es liegt nahe, dies als Signatur auf die Gedichte zu beziehen. Milnor gibt zu bedenken, dass nicht klar ist, in welcher Weise das Wort epoese sich in diesem spezifischen Kontext auf die Texte beziehen könnte. Insbesondere ist es fraglich, ob damit das Dichten oder der Vorgang des Schreibens gemeint ist. ${ }^{504}$ Nichtsdestotrotz werden diese Graffiti schon lange Tiburtinus als Individuum zugeschrieben, sodass diesem bisher als einzigem Pompejaner der Status eines Dichters zugesprochen wird. ${ }^{505}$ Aber auch Gigante ist vorsichtiger und bezieht die Signatur nur auf das erste Gedicht CIL IV 4966, dem die Signatur am nächsten zugeordnet ist. ${ }^{506}$

Bei einer ganzen Reihe weiterer Graffiti scheint dieser Bezug klarer zu sein. In CIL IV 8229 findet sich wie in einigen anderen das Wort scribere: amat qui scr(ibit). ${ }^{507}$ Wenn sich Tiburtinus epoese auf die nebenstehenden Epigramme bezieht, unterscheiden sich diese Angaben besonders auch durch die intradiegetische Beziehung zwischen dem „erzählten“ Autor und der Handlung am Text. Auch nicht sicher ist, ob die Personen ihren tatsächlichen Namen angegeben haben, ist doch deutlich zu erkennen, dass hier das Verfassen und Anbringen eines Graffito als Tat einer bestimmten Person gekennzeichnet werden sollte. ${ }^{508}$ Damit verbunden ist die Frage nach dem Status des Autors im Rahmen der pompejanischen Inschriften, die insbesondere Milnor aufwirft. Dipinti und Steininschriften wurden in der Regel mit Sicherheit von professionellen

501 Vgl. zu Angabe der Fundumstände und der Anbringungsstelle die Schede im CIL, Fiorelli 1883, 52 und Milnor 2014, 142.

502 Vgl. Morelli 2000, 239; Kruschwitz 2008, 253-253.

503 Vgl. aber: Buecheler 1883 und Cugusi 2007, 113. 172-173. 175-176.

504 Vgl. Milnor 2014, 147-149.

505 Vgl. Fiorelli 1883, 52-53; Della Corte 1926, 127.

506 „Е un arbitrio considerare Tiburtino autore anche degli altri testi, anche se possa essere suggestivo supporre un ciclo poetico e dar consistenza alla figura di un poeta pompeiano.“ Gigante 1979, 81. 84-85.

507 Ähnlich z. B. auch: CIL IV 8891: venimus hoc cupidi scribitt(!) Cornelius Martialis; CIL IV 1841: quisquis es amissos hin[c iam ob]/liviscere graios / scribit Narciss(us).

508 Milnor 2014, 148: „the desire to use a name to authorize the act of graffiti-writing is by no means unique to Tiburtinus“. 
Steinmetzen oder Malern vor allem handwerklich umgesetzt, aber nicht sprachlich formuliert. ${ }^{509}$ Bei Graffiti ist meist gerade dies der Fall und wird immer wieder als ein Charakteristikum betont. ${ }^{510}$ Während auf der einen Seite Zahlenreihen oder Datumsangaben, die inhaltlich kaum Varianz zuließen, vergessen lassen, dass sie von einem oder mehreren Individuen angebracht wurden, war bei den komplexeren Zeichnungen, den teilweise auch obszönen Verse und besonders kunstvolleren Epigrammen die Angabe eines oder mehrerer Urheber offenbar von Bedeutung.

\subsection{3 admiror te paries}

Drei Graffiti in Pompeji, die inhaltlich auf ein gemeinsames Vorbild zurückgehen müssen, können als Kommentare zur Praxis des Graffitischreibens bezeichnet werden. Im Zentrum steht hier die Frage nach der Rezeption und der Bewertung dieser Praxis durch die Zeitgenossen. Gerade dieser Aspekt ist aufgrund der Diversität des Materials und der Ubiquität der Graffiti nur schwer zu greifen. Bisherige Ergebnisse deuten darauf hin, dass es weitgehend nicht nur geduldet, sondern sogar willkommen war, die Wände von öffentlichen und privaten Gebäuden als Beschreibfläche zu nutzen. Die Texte der Inschriften (Abb. 32. F49) lauten: ${ }^{511}$

CIL IV 1904 (basilica): admiror o pariens(!) te non cecidisse ruinis qui tot / scriptorum taedia sustineas

CIL IV 2461 (Großes Theater): admiror te paries / te non cecidise(!) ruina

CIL IV 2487 (Amphitheater): admiror te paries non cecidisse / qui tot scriptorum taedia sustineas

Die Inschriften wurden innerhalb des antiken Stadtgebietes an weit voneinander entfernten Stellen in den Wandputz dreier öffentlicher Gebäude geritzt. Möglicherweise ist ein weiterer Graffito dieser Gruppe zuzurechnen, von dem sich jedoch nur das Wort admiror erhalten hat. ${ }^{512}$ Aus den apographa im CIL bzw. den erhaltenen Graffiti wird deutlich, dass alle drei (oder vier) von unterschiedlichen Händen geschrieben wurden. ${ }^{513}$ An oder in den Gebäuden, an denen sie angebracht waren, befanden sich jeweils weitere Graffiti. Der öffentliche oder halböffentliche Charakter dieser Räume spielte offenbar sowohl bei der Anbringung als auch bei der Wahrnehmung der Epigramme eine große Rolle: CIL IV 1904 befand sich an der nördlichen Innenwand der basilica am forum, CIL IV 2461 an der Außenseite der östlichen Begrenzung der scaena

509 Bei den formelhaften programmata und edicta munerum ist es jedoch sehr wahrscheinlich, dass die Schreiber in der Lage waren, die gängigen Formulierungen anhand der grundlegenden Informationen selbst zusammenzusetzen, falls sie dazu keine genauen Vorgaben erhalten hatten.

510 Z. B. Voegtle 2012, 106.

511 Alle drei auch: CLE 957.

512 CIL IV 1906.

513 Vgl. Franklin 1991, 82. 


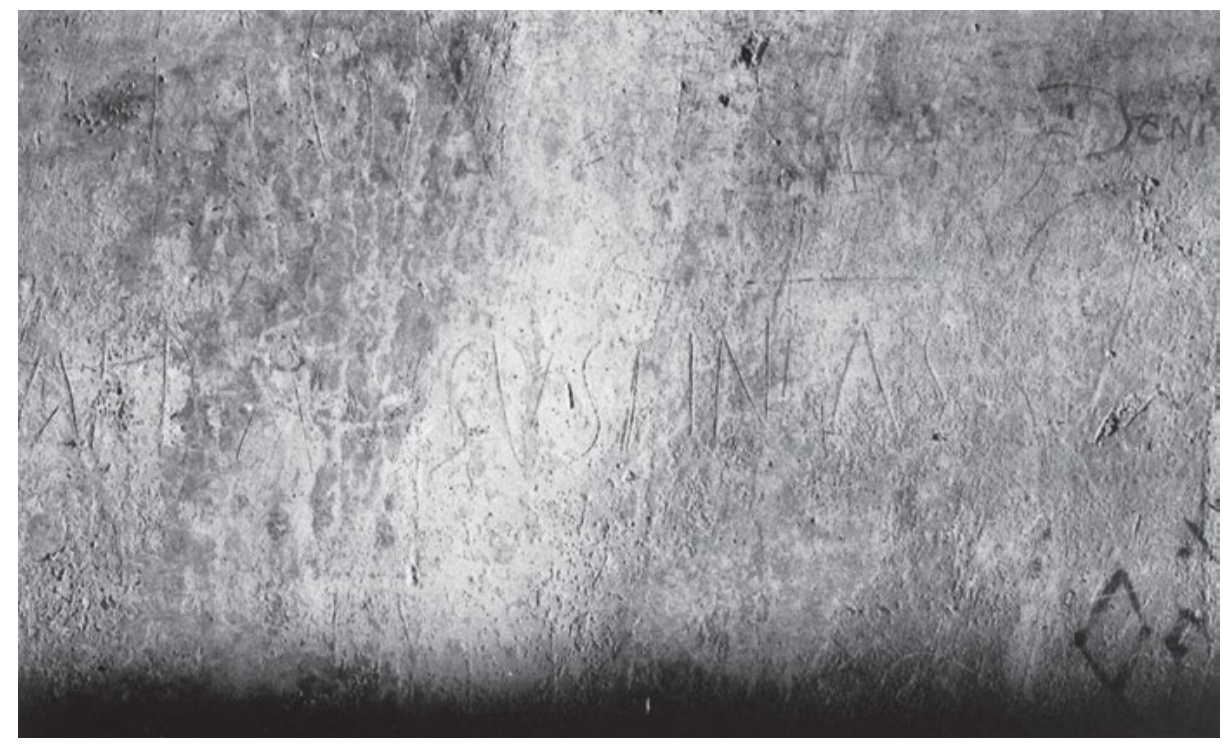

Abb. 32: CIL IV 2487 aus der crypta des Amphitheaters, die von der nördlichen Rampe nach Osten wegführt. @ Su concessione del Ministero per i Beni e le Attività Culturali e per il Turismo - Parco archeologico di Pompei. Jegliche weitere Reproduktion oder Duplikation ist untersagt.

des großen Theaters und CIL IV 2487 in der crypta des Amphitheaters. ${ }^{514}$ Keine der Formulierungen ist identisch, aber alle drei sind als Varianten desselben Motivs zu erkennen. CIL IV 1904 ist ein vollständiges Distichon, während CIL IV 2461 nur den Hexameter bietet, der bei CIL IV 2487 wiederum missglückt ist. ${ }^{515}$ Gerade in der basilica wurden zahlreiche weitere Versinschriften gefunden, in die sich dieser Graffito einreiht. ${ }^{516}$ Die Formulierung findet sich zwar nur in Pompeji, aber ähnliche Gedan-

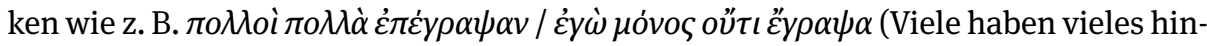
geschrieben, nur ich habe nichts geschrieben.) sind zum Beispiel in Rom in der Domus Tiberiana auf dem Palatin überliefert. ${ }^{517}$

Diese Graffiti sind keine Metatexte, die von Produktion und Rezeption eines bestimmten Textes berichten, sondern sie beziehen sich auf eine ganze Textgruppe. Diese Gruppe wird vor allem über den Anbringungsort definiert: die Wand. Sie sind

514 Die Putzfläche, in die CIL IV 1904 eingeritzt ist, wurde abgenommen und befindet sich heute im Museo Archeologico Nazionale di Napoli (Inv. 4706). CIL IV 2461 ist verloren, CIL IV 2487 ist am Anbringungsort erhalten.

515 Zum Metrum: Kruschwitz 2009, 503. Zur Textverteilung Vgl. Anm. 382.

516 Vgl. Milnor 2014, 30-31 betont, dass der Graffito je nach Kontext Bedeutungsverschiebungen erfahren konnte; CIL IV 1780-1947.

517 Castrén/Lilius 1970, 145 Nr.70; Taf. 31 und 32. Der Graffito befindet sich ebenfalls auf einer dicht beschriebenen Wand. Vgl. auch Langner 2001, 21. Zu dem griechischen Graffito: 126-127 mit Abb. 69; $\mathrm{Zu}$ weiteren vergleichbaren Zeugnissen: Zangemeister in CIL IV Hauptband S. V. 
vordergründig nicht selbstreferentiell, sondern nehmen Bezug auf andere, reale Artefakte, die als taedia bezeichnet werden, und sind von zahlreichen ähnlichen Wandinschriften umgeben. Das Versmaß des Distichon, also die Kombination von Hexameter und Pentameter ist ebenso wie der reine Hexameter in Pompeji häufig belegt. Auch die Gestaltung der Zeilen und einzelnen Buchstaben hebt diese Texte nicht von anderen ab. Sie sind wie viele andere Graffiti in einer Mischung aus Kursiven und nichtKursiven geschrieben. Ebensowenig sind die Verschreibungen pariens statt paries und cecidise statt cecidisse ungewöhnlich. ${ }^{518}$ Sie sind demnach von denselben Mustern geprägt und haben grundsätzlich keinen anderen Status als die Texte, auf welche sie sich despektierlich zu beziehen scheinen. Da sie somit der gleichen Gattung angehören wie die taedia, die sie anprangern, ist es fraglich, inwieweit sie tatsächlich kritisch gegenüber diesen Stellung beziehen konnten.

Dennoch ermöglichen sie Rückschlüsse auf die Rolle und Rezeption von Geschriebenem zunächst auf einer ganz grundlegenden Ebene: Graffiti wurden offenbar auch in dunkleren Bereichen, wie dem Gang im Amphitheater gesehen und als zahlreich wahrgenommen. Das Schreiben solcher Texte wird als eine Praxis dargestellt, die von vielen Personen ausgeübt wurde. Eine mögliche Form der Rezeption von Graffiti bestand darin, selbst einen Text hinzuzufügen, wie es hier der Fall war. Im Bezug auf andere soziale Felder, in denen das Schreiben auf die Wände eine Rolle spielte, ist es besonders interessant, dass auch Personen, die Graffiti schrieben, als scriptores bezeichnet wurden. ${ }^{519}$ Der Vermerk scripsit, der bei einigen Dipinti vorkommt, hätte ansonsten vermuten lassen, dass es sich um eine Art Berufsbezeichnung der professionellen Schreiber handeln könnte. ${ }^{520}$ Soweit scheint die Deutung unstrittig.

Zentral für eine weitergehende Deutung ist die Formulierung tot scriptorum taedia. Zunächst kann festgehalten werden, dass sich darin die Möglichkeit oder das Bewusstsein ausdrückt, dass manche Leute die Präsenz von Graffiti - sei es aus inhaltlichen oder aus optischen Gründen - als störend empfinden könnten. Immer wieder wurde angenommen, dass auch der Autor und Schreiber diese Meinung teilte. ${ }^{521}$ Dies ist jedoch aus verschiedenen Gründen auszuschließen. Erstens erscheint der Zweizeiler wie ein lateinisches Pendant des oben genannten griechischen Textes und gibt somit ein bekanntes Motiv wieder. Zweitens ist die übertrieben pathetische Formulierung (o paries) und die Wahl der metrischen Form, insbesondere mit zwei Spondeen

518 Keegan weist zudem darauf hin, dass das $<\mathrm{I}>$ in admiror durch die Position kurz ist, obwohl es in diesem Wort natürlicherweise lang sein müsste: Keegan 2011, 174. Zu cecidise und necese statt cecidisse und necesse vgl. Väänänen 1937, 104; zu pariens statt paries vgl. Väänänen 1937, 119.

519 Vgl. dazu: Kruschwitz 2010, 216.

520 Vgl. Chiavia 2002, 86 Anm.137. Gigante jedoch bezieht das ganze Gedicht auf die programmata und somit auch den Begriff scriptor auf die professionellen Schreiber. Da allerdings in keinem der Fundkontexte gemalte Inschriften vorkamen, ist es unwahrscheinlich, dass der jeweilige Schreiber mit taedia Dipinti meinte. Gigante 1979, 233. Vgl. auch Magaldi 1930, 51.

521 Als ernst gemeintes, negatives Qualitätsurteil wird das Gedicht aufgefasst von: Mau 1908, 510511; Väänänen 1937, 17; Solin 1979, 278-279; Franklin 1991, 82; Varone 1993, 41. 
in der ersten Hälfte des Pentameter entlarvend. ${ }^{522}$ Die Graffiti sind demnach auf der einen Seite als Metatexte, also als Geschriebenes über Geschriebenes, inszeniert. Auf der anderen Seite gleichen sie überdeutlich den Texten, von denen sie handeln: in formaler und gestalterischer Hinsicht, aber auch durch die Wahl der Anbringungsstelle. Dadurch, dass sie in der Nähe von anderen Graffiti an die Wände geschrieben wurden, werden sie überhaupt erst zu Kommentaren über diese. ${ }^{523}$ Mit dieser formal wie inhaltlich beschreibbaren Verschränkung oder mise en abyme werden sie einerseits Teil der Graffitilandschaft und heben sich andererseits von ihr ab. ${ }^{524}$ So heben sie ihren eigenen Aussagewert einerseits auf - man könnte sie als fingierte Metatexte bezeichnen. Andererseits reflektieren sie den Status des „Geschriebenen über Geschriebenes“, indem sie sich selbst als unseriös entlarven und werden dadurch zu Meta-Metatexten. ${ }^{525}$ Letztendlich entlarven sie sich nicht nur selbst, sondern machen vermittels der Wahl ihres Anbringungsortes und der Übertreibung der literarischen Form auch mögliche ernst gemeinte Kritik lächerlich.

\subsubsection{Graffiti mit besonderer Reflexion über die Gestaltung}

Eine Reihe von Graffiti weist auf eine intensive Reflexion der gestalterischen Merkmale von Buchstaben und Wandinschriften hin. Sie zeichnen sich alle dadurch aus, dass sie textuell bruchstückhaft sind oder nur aus einzelnen Buchstaben bestehen. Die Buchstaben selbst wurden jedoch vergleichsweise aufwendig gestaltet. Es handelt sich um die Graffiti CIL IV 4119, 8388a-d, 8893 und 10203b sowie einen weiteren unpublizierten Graffito. Besonderes Interesse brachten die Urheber dieser Graffiti der Konstruktion der Buchstaben entgegen. ${ }^{526}$

In CIL IV 10203b (STQRVS) zeigt sich deutlich, dass der Schreiber die Monumentalform der Schrift nachahmen wollte (Abb.33). Dies verfolgte er nicht nur durch eine doppelte Linienführung, sondern auch durch eine Unterscheidung zwischen Haarund Schattenstrich sowie Serifen, die besonders deutlich am Schaft des T und am V zu erkennen sind. Noch weiter ging der Schreiber von CIL IV 4119 (Primus hic) (Abb. 34). Auch er gestaltete die Umrisse mit Haar- und Schattenstrich und Serifen. Zudem schraffierte er jedoch die so umrissenen Bereiche, sodass sich auch diese Flächen

522 So deutet auch Langner 2001, 21. Milnor bemerkt dazu, dass manche Inschriften sich anscheinend ihrer selbst als Teile eines größeren Textcorpus bewusst seien: Milnor 2014, 29: „some graffiti texts have a sense of themselves as part of a larger written genre“ und „the couplet was obviously created to be written on a wall“.

523 Vgl. Milnor 2014, 30-31.

524 Vgl. Zadorojnyi 2011, 117.

525 Die Mischung, die in diesem Zweizeiler formal und inhaltlich aufgebaut wird, wird auch von Keegan sehr überzeugend herausgearbeitet: Keegan 2011, 174.

526 Zu diesen Graffiti vgl auch: Lohmann 2018, 262-264. 


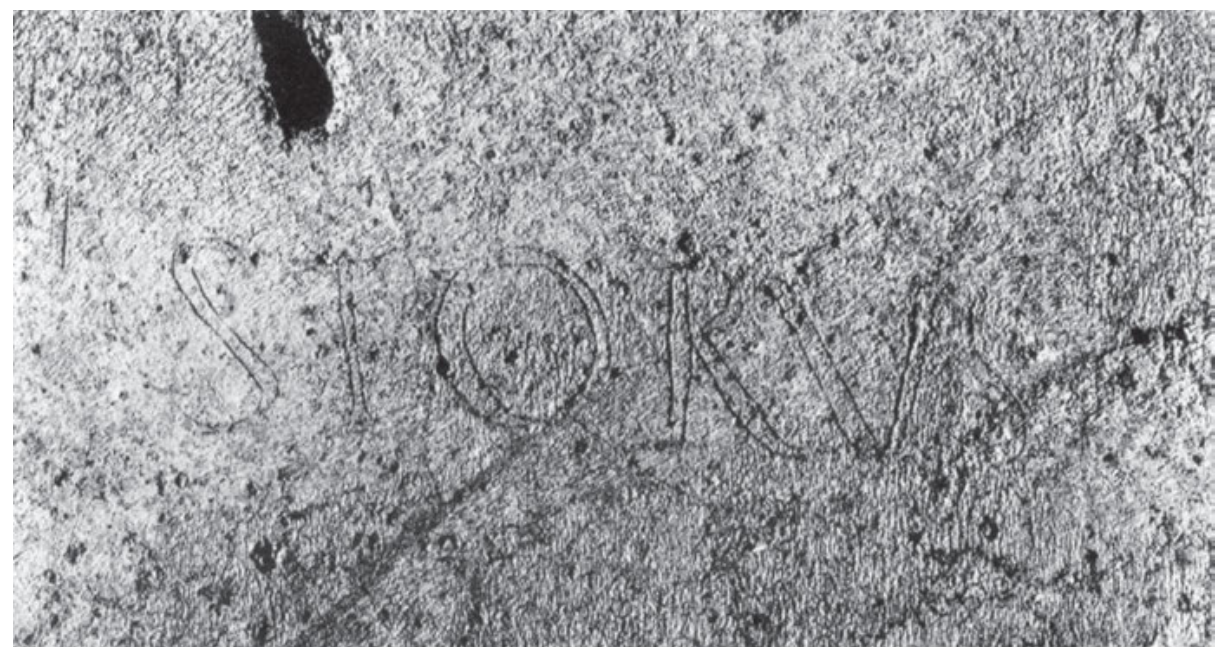

Abb. 33: CIL IV 10203b in taberna VI 16, 33. @ Su concessione del Ministero per i Beni e le Attività Culturali e per il Turismo - Parco archeologico di Pompei. Jegliche weitere Reproduktion oder Duplikation ist untersagt.

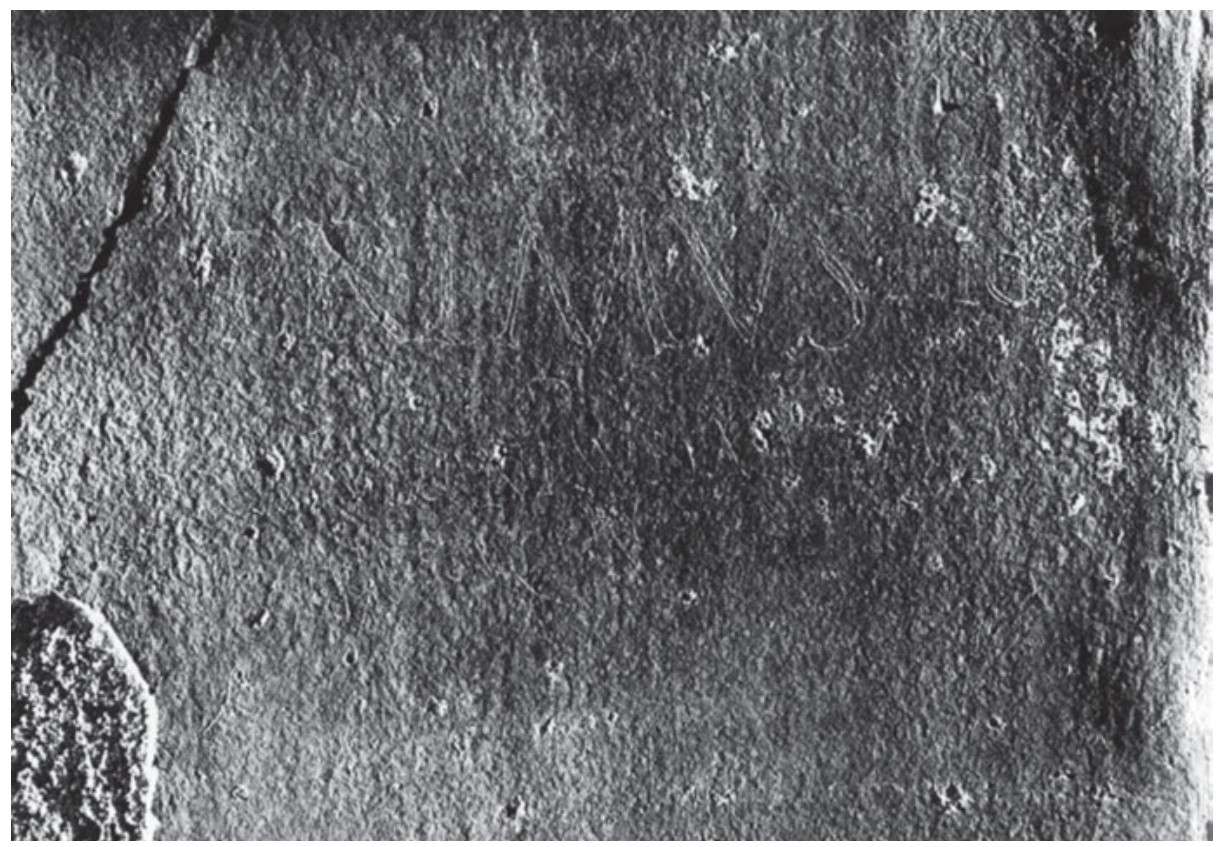

Abb. 34: CIL IV $4119 \mathrm{im}$ peristylium $\mathrm{p}$ von V 2, 4. ( S Su concessione del Ministero per i Beni e le Attività Culturali e per il Turismo - Parco archeologico di Pompei. Jegliche weitere Reproduktion oder Duplikation ist untersagt. 

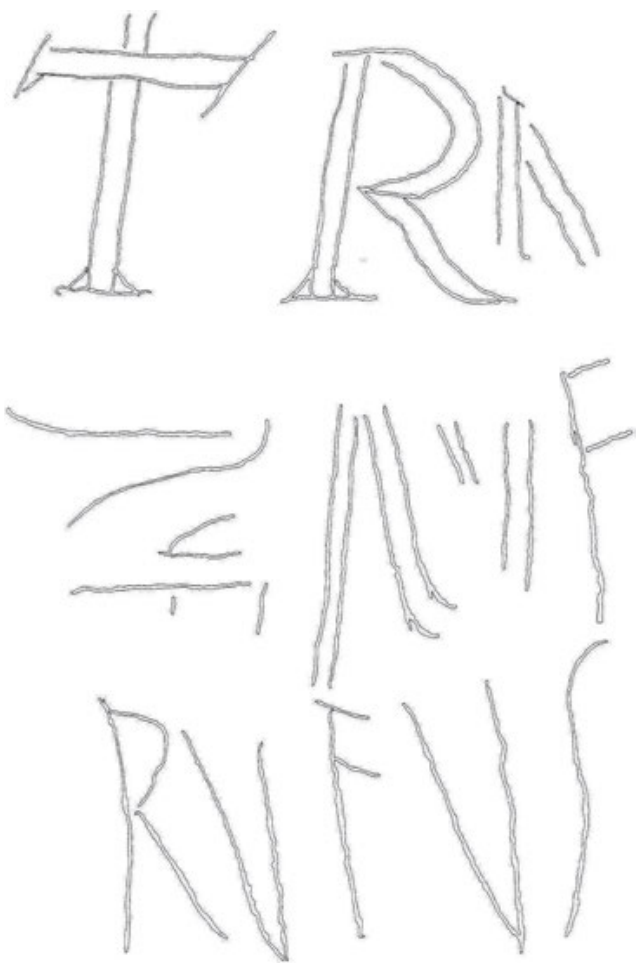

Abb. 35: CIL IV 8388 in Raum 3 von I 10, 8. (C) Su concessione del Ministero per i Beni e le Attività Culturali e per il Turismo Parco archeologico di Pompei. Jegliche weitere Reproduktion oder Duplikation ist untersagt.

wie bei gemalten und gemeißelten Inschriften qualitativ vom Umfeld absetzten. Bei CIL IV 8388, 8893 und dem $B$ ist dies nicht geschehen. Doch die Schreiber dieser Inschriften stellten die Buchstaben nicht einfach als Umrisse dar, sondern konstruierten diese aus mehreren Zügen. So sind beim $T$ und beim $R$ von $8388 \mathrm{c}+\mathrm{d}$ jeweils mehrere Züge sowie die Serifen voneinander abgesetzt (Abb. 35). Ähnlich verfuhr der Schreiber des oben erwähnten Buchstabens $B$ (Abb.36), doch er schuf sich weitere Hilfen bei der Konstruktion des Buchstabens, indem er ein Trapez vorzeichnete, welches die Höhe des Buchstaben und - nach dem 2. Strahlensatz - ein regelmäßiges Verhältnis von Höhe und Breite der beiden Bögen zueinander vorgab. Anscheinend ging es ihm vorrangig darum, dieses Konstruktionsprinzip zu erarbeiten oder darzustellen und erst in zweiter Linie um die Ästhetik des fertigen Buchstaben, da zum Beispiel die Schwellung am unteren Bogen recht nachlässig ausgeführt wurde. Zuletzt bietet auch CIL IV 8893 (L(uci?) V(aleri?) / L(uci?) Valer(i?)) interessante Einblicke in die Sicht eines Schreibers auf die Buchstabenformen, mit denen er sich in seinem Umfeld konfrontiert sah (Abb. 37). Die Umrisse entsprechen einer relativ breit angelegten actuaria. Darüber hinaus besteht die Besonderheit dieser Inschrift aber darin, dass der Schreiber die Buchstabenformen nicht zunächst als Umrisse darstellte und diesen dann in ihre Bestandteile unterteilte wie bei CIL IV 8388d, also zum Beispiel Serifen als kleine Keile an die Stämme ansetzte. Stattdessen führte er die Buchstaben 


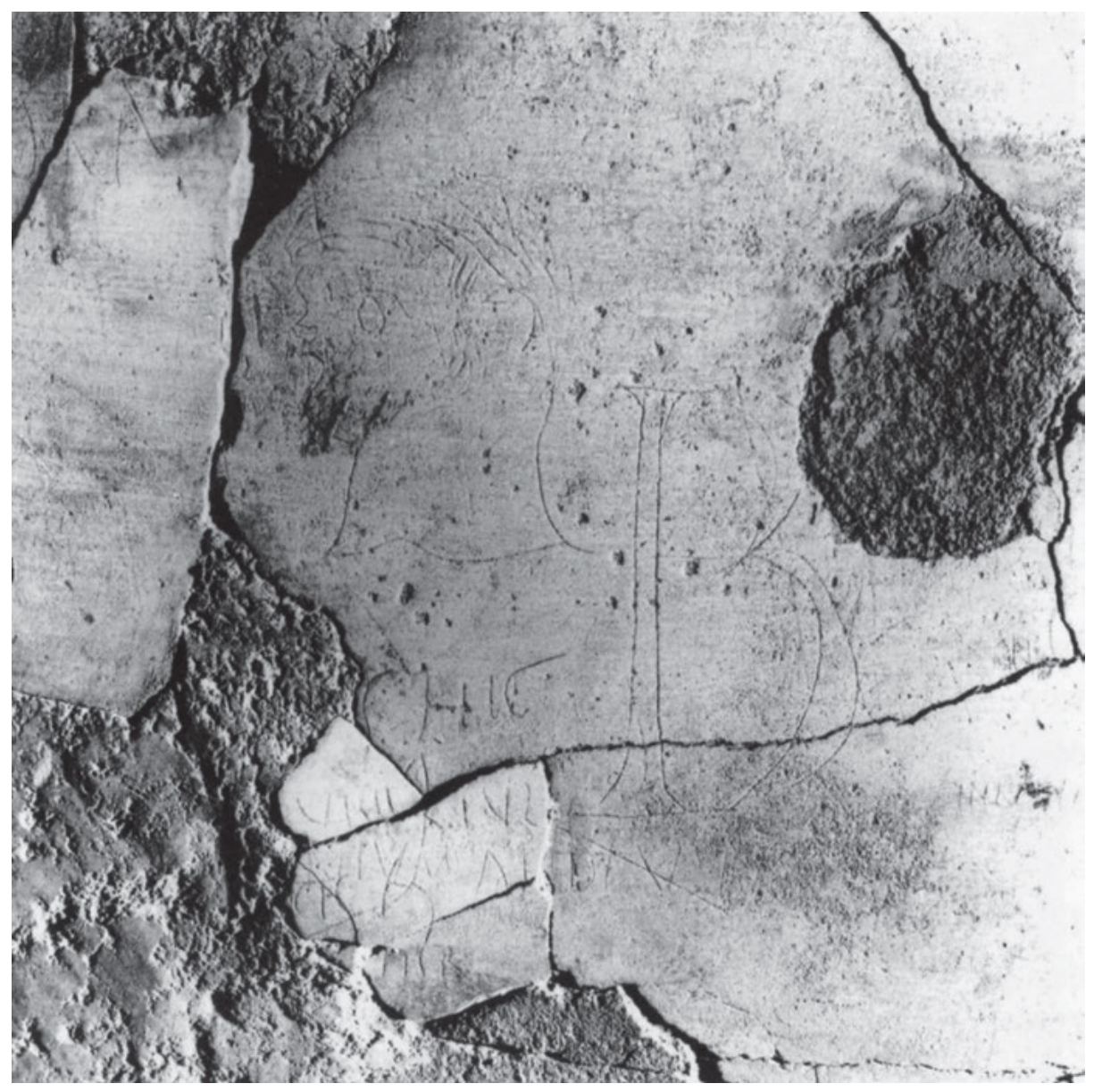

Abb. 36: Buchstabe B neben CIL IV 1854a aus der basilica, heute im Museo Archeologico Nazionale di Napoli Inv. 4684. (C) Su concessione del Ministero per i Beni e le Attività Culturali e per il Turismo - Museo Archeologico Nazionale di Napoli. Jegliche weitere Reproduktion oder Duplikation ist untersagt.

in mehreren durchgehenden Zügen aus - als ob er mit einem Pinsel malte. Dabei verwendete er vermutlich einen doppelten stilus, wie am Anstrich und am Scheitel des $V$ aber auch an den strikt parallel verlaufenden Linien aller Buchstaben gut zu erkennen ist. Bei der spitz zulaufenden Serife des $L$ muss er jedoch entweder den Doppelgriffel gekippt oder nachträglich einen kurzen Strich ergänzt haben. Bei Dipinti können diese spitz zulaufenden Enden dagegen mit einem flachen, aber weichen Pinsel völlig problemlos erzielt werden, da dabei nicht nur der Winkel, sondern auch der Druck verändert werden kann. ${ }^{527}$

527 Dazu Catich 1968, 45. 182-183. 


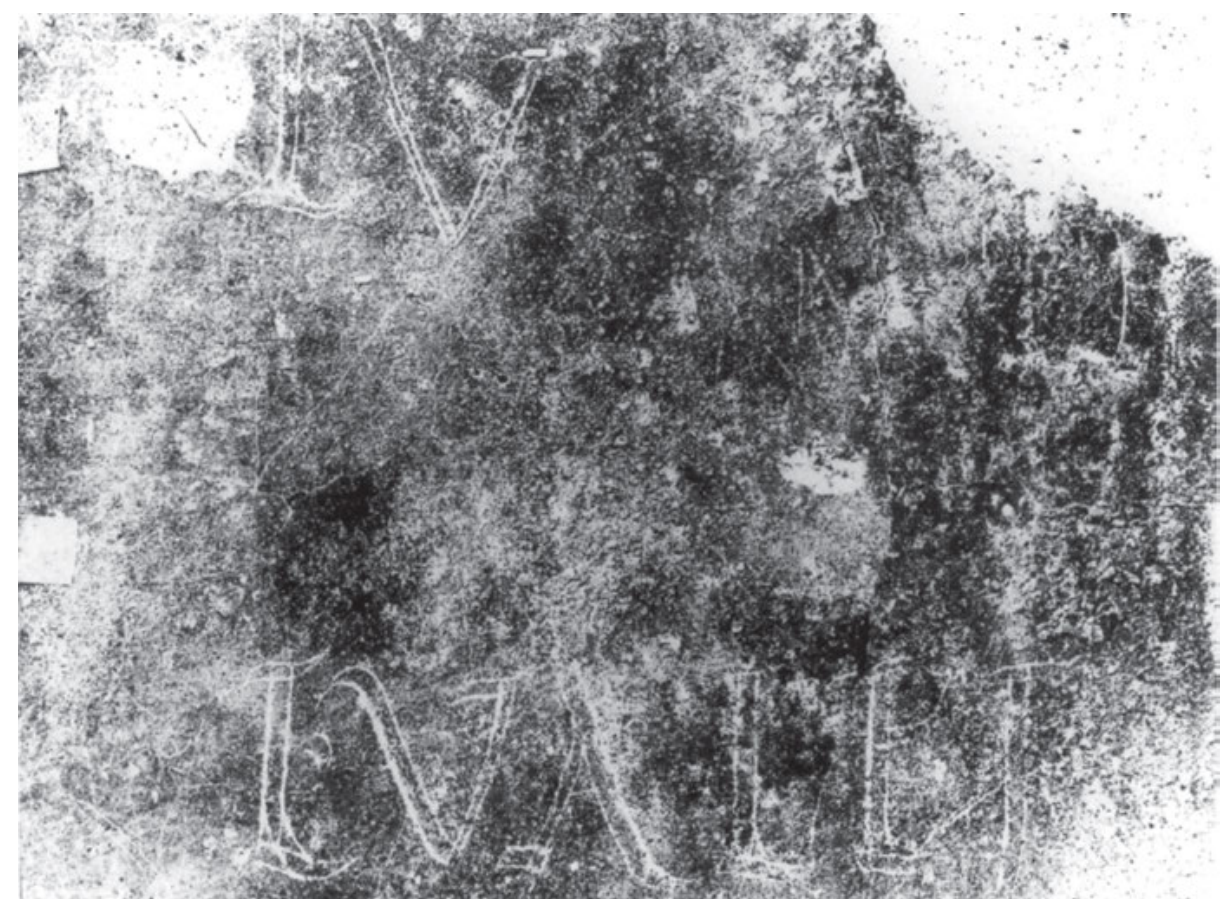

Abb. 37: CIL IV 8893 rechts von Eingang III 5, 3. (C) Su concessione del Ministero per i Beni e le Attività Culturali e per il Turismo - Parco archeologico di Pompei. Jegliche weitere Reproduktion oder Duplikation ist untersagt.

Es ist sehr unwahrscheinlich, dass die Schreiber hier in einem zweiten Schritt die Linien in ihrer ganzen Breite ausmeißeln wollten, auch wenn in Pompeji neben dem schraffierten Graffito auch drei weitere Beispiele für eine flächigere Ausgestaltung zu finden sind: CIL IV 1874 und 10030 sowie der rot ausgemalte Dipinto CIL IV 1372. ${ }^{528}$ Bei allen Beispielen spielte sicherlich sowohl ein Ausloten der Gestaltungsvarianten als auch eine Darstellung der eigenen Fertigkeiten eine große Rolle. Insbesondere bei CIL IV 8893 ist zu vermuten, dass es sich um einen sehr geübten, wenn nicht gar professionellen Schreiber handelte. Vor diesem Hintergrund sei auch noch einmal darauf hingewiesen, dass Ritzungen auch bei der Gestaltung und Fertigung von Steininschriften eine große Rolle spielten. ${ }^{529}$

528 Abbildungen: Varone 2012, 371. 90-91. 265. Beispiele für Graffiti, die ähnlich angelegt sind, und ausgemeißelt wurde, finden sich aber z. B. unter denen im paedagogium auf dem Palatin: Solin et al. 1966, Nr. 284 S. 221; Nr. 291 S. 224; Nr. 347 S. 241; Nr. 352 S. 243; Nr. 367 S. 248.

529 Dazu grundlegend: Mallon 1955. 


\subsection{Bildliche Darstellungen}

In Pompeji wurden zwei Bilder gefunden, die Schrift im öffentlichen Raum zeigen: der Fries aus dem atrium 24 der praedia Iuliae Felicis (II 4, 3) und ein Gemälde aus der Casa della Rissa nell'Anfiteatro (I 3, 23), welches dort an der Westwand des Peristyls zu sehen war. ${ }^{530}$ Beide Malereien befinden sich mittlerweile im Museum in Neapel. ${ }^{531}$ Zudem sind vor allem aus Pompeji Schrift-Bild-Kombinationen bekannt, in denen Schrift durch spezifische Gestaltungsmittel eingerahmt und aus der Masse an Inschriften herausgehoben wird.

\subsubsection{Dipinti im Gemälde aus der Casa della Rissa nell’Anfiteatro}

Das Gemälde aus der Casa della Rissa nell'Anfiteatro (Abb. 3.10) war an der westlichen, geschlossenen Wand des Rumpfperistyls oder Hofes angebracht. Es ist $170 \mathrm{~cm}$ hoch und $185 \mathrm{~cm}$ breit. Der Hof war im Osten und von Norden durch Säulen gerahmt, wobei diese an der Nordseite durch niedrige Mauern verbunden wurden. An der Südseite hatte man die ehemalige porticus zugemauert, sodass der Bereich dahinter völlig vom Hof abgetrennt war. Das Gemälde war daher vom Hof aus und von den Hallen im Osten und Norden sichtbar. ${ }^{532}$ Anders als das Gemälde selbst, können die Veränderungen im Peristyl- bzw. Hofbereich nicht datiert werden. Im ganzen Haus wurden zu einem oder mehreren nicht näher bestimmbaren Zeitpunkten Veränderungen vorgenommen..$^{533}$

Das Bild zeigt eine komplexe, vielfigurige Szene in einem größeren räumlichen Kontext. Es ist allgemein akzeptiert, dass es sich bei dem räumlichen Umfeld um das Amphitheater von Pompeji, den campus und Teile der Stadtmauer im Südosten der Stadt handelt, die von Norden aus der Vogelperspektive gesehen werden. Die Figuren, die in der arena, auf der cavea und in allen Bereichen zwischen den Gebäuden zu sehen sind, sind in Kampf-Konstellationen gezeigt. Aller Wahrscheinlichkeit nach ist hiermit die äußerst brutale und blutige Schlägerei zwischen den Einwohnern Pompejis und denen der Nachbarstadt Nuceria gemeint, die im Jahr 59 n. Chr. in Pompeji

$530 \mathrm{Zu}$ Bildern, die Schrift und Schreiben nicht in einem bestimmbaren räumlichen Kontext zeigen, zugleich aufgrund der dargestellten Utensilien eher in den privaten Bereich einzuordnen sind, vgl. vor allem: Meyer 2009.

531 Invv. 9068 (praedia Iuliae Felicis) und 112222 (Casa della Rissa nell’Anfiteatro).

532 Von den Räumen i und k, die nördlich an den Hofbereich anschlossen, war es nur dann zu sehen, wenn man direkt in der Tür stand.

533 Besonders auch im atrium-Bereich, wo man auf das benachbarte Grundstück übergriff. Vgl. Sampaolo 1990, 77-78. Das Haus wurde 1868 ausgegraben. Eine kurze Beschreibung findet sich auch in Fiorelli 1875, 55-56. 
ausgetragen wurde und in deren Folge ein Verbot ausging, das Gladiatorenspiele in Pompeji für zehn Jahre untersagte. ${ }^{534}$

Offenbar spielte die Identifizierbarkeit der Gebäude anhand signifikanter Merkmale eine große Rolle. Dafür wurden jedoch Ungenauigkeiten in Kauf genommen. So entspricht die Distanz zwischen Amphitheater und der östlich (im Bild links) in einigem Abstand dazu verlaufenden Stadtmauer nicht dem Befund, wo die cavea direkt an die Mauer anschließt. Auch für das kleine gemauerte Bauwerk im Vordergrund konnte bisher keine Entsprechung gefunden werden. Die charakteristische Treppenkonstruktion an der Außenseite des Amphitheaters wurde nach Norden gerückt, sodass sie vollständig zu sehen ist. Die Bepflanzung und die Eingänge des campus wiederum sind akkurat wiedergegeben, wie der Vergleich mit dem archäologischen Befund zeigt. ${ }^{535}$

An der Nordseite des im Gemälde dargestellten campus sind zwei Eingänge wiedergegeben. Oberhalb des linken Eingangs und unmittelbar an der Gebäudeecke ansetzend ist in lateinischen Buchstaben der folgende Text zu lesen: $D$ (ecimo) Lucretio Fel(!)citer. ${ }^{536}$ Etwas unterhalb und zwischen den beiden Eingängen konnte

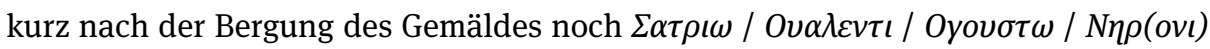
$\varphi \eta \lambda \iota \kappa \iota \varepsilon \varepsilon \rho^{537}$ gelesen werden. Obwohl sich der Zustand verschlechtert hat, können diese Lesarten bestätigt werden. Aufgrund der Position und der Farbe müssen hiermit Dipinti, die auf die Außenwand des campus gemalt waren, gemeint sein.

An der Nordseite des campus sind tatsächlich zwei Eingänge vorhanden. Westlich davon ist eine größere Putzfläche erhalten. Dort sind mehrere sehr großformatige Dipinti - programmata und edicta munerum - dokumentiert. ${ }^{538}$ An der Ecke und zwischen den beiden Eingängen fehlt der Putz. Es ist sehr wahrscheinlich, dass die gesamte Fassade als Beschreibfläche genutzt wurde, zum einen, weil auf der erhaltenen Oberfläche viele Inschriften gefunden wurden, und zum anderen, weil die Wand von allen Besuchern der Veranstaltungen im Amphitheater vor und nach den Spielen passiert werden musste.

Im Vergleich mit den erhaltenen Dipinti bringt dieser Befund zunächst keine zusätzlichen Erkenntnisse bezüglich der beteiligten Akteure, der Anbringungsorte, Themen oder Anlässe für die Anbringung von Dipinti. Grüße an hochrangige Mitbürger oder Angehörige des Kaiserhauses gehören zu den häufigeren Textsorten der

534 Vgl. Wallace-Hadrill 2011a, 297; Sampaolo 1990, 80-81 mit Abb.6a-6b. Zu der überlieferten Schlägerei: Tac. Ann. 14,17.

$535 \mathrm{Zu}$ den Details des Gemäldes und Beobachtungen im Verhältnis zum archäologischen Befund: I 3, 23 in PPM I (1990) 80-81; Pesando 2001, 186-188; Guzzo 2007, 142. Pietro Giovanni Guzzo weist mit Bezug auf die Einordnung der Malereien an der Innenseite der Brüstung rund um die arena, die chronologisch problematisch ist, darauf hin, dass hier wahrscheinlich eher allgemein auf eine freskale Gestaltung verwiesen wurde als auf ein konkretes Motiv.

536 CIL IV 2993x.

537 CIL IV 2993y. Heute ist vor allem die zweite Inschrift nicht mehr vollständig zu erkennen.

538 Vgl. Kapitel 4.2. 
Dipinti. ${ }^{539}$ Die genannten Namen der im Bild gezeigten Inschriften CIL IV 2993x und 2993y sind Decimus Lucretius, Satrius Valens und Augustus Nero. Sehr wahrscheinlich sind hier neben dem Kaiser nicht zwei, sondern lediglich eine weitere Person gemeint: Decimus Lucretius Satrius Valens, der auch aus anderen Inschriften bekannt ist. ${ }^{540}$ Er war flamen Neronis Caesaris Augusti fili, wodurch sich die Nennung zusammen mit dem Kaiser innerhalb der griechischen Inschrift erklären lässt. Es handelt sich also um drei Grüße an zwei Personen.

Was den Anbringungsort betrifft, wäre es theoretisch möglich, dass diese Inschriften tatsächlich dort zu lesen waren und daher vom Maler als Detail mimetisch wiedergegeben wurden. ${ }^{541}$ Dagegen spricht aber nicht nur die Konstellation der aufgeteilten Namen des Decimus Lucretius Satrius Valens, sondern auch der Umstand, dass ansonsten keine großformatigen Dipinti in griechischer Schrift aus Pompeji oder Herculaneum bekannt sind. Vielmehr scheint die Erklärung dafür, dass diese beiden Texte Teil des Bildes waren, auf inhaltlicher Ebene zu suchen zu sein. Da ganz eindeutig die Kämpfe das Hauptthema des Bildes sind, könnte auch die Inschrift im Zusammenhang mit der Rolle des Lucretius im Rahmen dieser und der darauf folgenden Vorgänge stehen. Sabbatini Tumolesi nimmt an, dass er, nachdem das Verbot ergangen war, seine guten Beziehungen zum Kaiser einsetzte, um eine Milderung der Strafe zu erwirken. ${ }^{542}$ Eine solche Aufhebung der Strafe wäre in Anbetracht der Tatsache, dass die Ausschreitungen nach Tacitus' Bericht äußerst gewaltsam waren und dass viele Menschen starben, allerdings überraschend. ${ }^{543}$ Die explizite Darstellung der Kämpfe im Gemälde legt zudem den Fokus offensichtlich auf die Ausschreitungen und ist nicht als eine verharmlosende Rückblende anzusehen. Fabrizio Pesando nimmt daher an, dass die Inschriften eher als ein Hinweis darauf, dass gerade kein Widerstand gegen die Beschlüsse geleistet wurde und dass die feliciter-Wünsche an den Kaiser und an einen Pompejaner, der in enger Beziehung zu ihm stand, eher als Zeichen der Annahme der Strafe zu deuten sind. ${ }^{544}$ Ob der Hausbesitzer in einem persönlichen Verhältnis zu Lucretius stand oder ob er ihn lediglich als einen der prominentesten Ausrichter von Unterhaltungsveranstaltungen ausgewählt hat, ist nicht zu klären. Die

539 Ein weiteres Beispiel ist ein Gruß an Cnaeus Alleius Nigidius Maius an den praedia Iuliae Felicis CIL IV 7990. CIL IV 7996: flamini Neronis Caesaris feliciter könnte sich ebenfalls auf Decimus Lucretius Satrius Valens beziehen.

540 Insbesondere aus den beiden edicta munerum CIL IV 7992 und 7995.

541 Vgl. Pesando 2001, 188.

542 Sie sieht mit Blick auf diese Deutung das Gemälde nicht negativ, als Darstellung der Gewalt, sondern positiv, als eine Feier der Wiederaufnahme der Spiele, die dem Einsatz des Lucretius zu verdanken war Sabbatini Tumolesi 1980, 25-26.

543 Sub idem tempus levi contentione orta atrox caedes inter colonos Nucerinos Pompeianosque gladiatorio spectaculo [...]. ergo deportati sunt in urbem multi e Nucerinis trunco per vulnera corpore, ac plerique liberorum aut parentum mortes deflebant. (Tac. Ann.14,17,1) Zitiert nach P. Corneli Taciti libri qui supersunt edidit Koestermann tom. I ab excessu divi Augusti (Leipzig 1960).

544 Vgl. Pesando 2001, 188. 
Auswahl des Inhaltes und die Doppelung in griechischer und lateinischer Schrift findet darüber hinaus weitere Parallelen innerhalb dieses Hauses. Der Themenbereich des Amphitheaters war dem Besitzer von Haus I 3, 23 offenbar sehr wichtig. Neben dem hier besprochen Bild und darunter, auf einer älteren Putzschicht, wurde weitere Gladiatorendarstellungen gefunden, die jedoch alle verloren sind. ${ }^{545}$ Auch bei diesen fanden sich Beischriften in griechischer Schrift, sodass diese Besonderheit der am campus im Gemälde dargestellten Inschriften vermutlich eher auf die Herkunft oder Bildung des Hausbesitzers zurückzuführen ist als dass sie tatsächliche griechische Dipinti wiedergeben sollte.

Mit Blick auf die Wahrnehmung der gemalten Wandinschriften ist es bedeutsam, dass gerade dieser Teil des Gemäldes als Anbringungsstelle von Inschriften dargestellt wird. Wie erwähnt fanden sich an der Nordwand des campus zahlreiche Inschriften. Darüber hinaus entspricht die Größe der im Bild gezeigten Dipinti den Proportionen der im Befund dokumentierten Inschriften, die teilweise bis $\mathrm{zu} 70 \mathrm{~cm}$ hohe Buchstaben aufweisen. Diese müssen für einen Betrachter, der von einer der Querstraßen der Via dell'Abbondanza her kam, eine ähnliche Fernwirkung erzeugt haben, wie sie in der Konstellation von Architektur und Schrift im Gemälde suggeriert wird. Es handelt sich somit einerseits um eine plausible Darstellung der Wandinschriften. Andererseits wird es deutlich, dass die Dipinti an der Nordseite des campus in ihrer Position und Größe als so charakteristische Kennzeichen des Gebäudes empfunden wurden, dass sie bei der detaillierten Schilderung eines historischen Geschehens eine Rolle spielten. Wandinschriften waren visuelle Merkmale dieses Teilbereiches der Stadt. In dem Gemälde werden somit Konstellationen aus der Realität mit gezielten Bezügen auf einzelne Personen kombiniert.

\subsection{2 tabulae dealbatae im Fresko aus den praedia Iuliae Felicis}

Der Fries aus dem atrium (Raum 24) der praedia Iuliae Felicis (II 4, 3) ist besonders deshalb interessant, weil in ihm eine Inschriftengattung gezeigt wird, die ansonsten nur aus literarischen Quellen bekannt ist: wir erfahren durch sie etwas über die Anbringung, die Gestaltung und die Rezeption von tabulae dealbatae im öffentlichen Raum.

Teile dieses Anwesens wurden bereits in der Zeit zwischen 1755 und 1757 ausgegraben. ${ }^{546}$ Ein umlaufender Fries wurde aus dem atrium geborgen, in einzelne Partien unterteilt und gerahmt. ${ }^{547}$ Heute befindet er sich zum größten Teil im Museum

545 Sampaolo 1990, 78.

546 Dazu ausführlich: Parslow 1988 und Parslow 1996 sowie Olivito 2013, 13-35. Vgl. Fiorelli 1860, 12-54; (ohne Autor) 1762, 226-227 Taf. 43.

547 Zur Entdeckung des Frieses Ende Mai bzw. Anfang Juni 1755: Fiorelli 1860, 17-18; Olivito 2013, $23-25$. 
in Neapel. ${ }^{548}$ Die Malerei zeigt im Vordergrund zahlreiche Monumente und Figuren, die in unterschiedliche Aktivitäten involviert sind. Den Hintergrund bildet eine zweigeschossige Säulenstellung. ${ }^{549}$ Die neuzeitliche Unterteilung und Rahmung unterstreicht den szenenartigen Charakter oder erzeugt möglicherweise überhaupt erst den Eindruck, dass es sich um geschlossene Szenen handelt. ${ }^{550}$ Auf den einzelnen Fragmenten sind Verkaufsszenen, Gruppen von Personen, Tiere, ein Fuhrwerk, Handwerksszenen und weitere Aktivitäten zu erkennen. Dieses Spektrum führte zusammen mit dem architektonischen Hintergrund der zweigeschossigen Säulenstellung dazu, dass die Malerei als Darstellung des forum von Pompeji gedeutet wurde. ${ }^{551}$ Kritiker dieser These führen vor allem Widersprüche mit dem archäologischen Befund ins Feld. So konnten Fuhrwerke den Platz zumindest zuletzt nicht befahren und die Gestaltung der Säulen entspricht nicht den noch vorhandenen Überresten. ${ }^{552}$ Guzzo nimmt deshalb an, dass zwar ein forum, aber nicht spezifisch das von Pompeji gemeint sei. ${ }^{553}$ Riccardo Olivito argumentiert dagegen, dass Details wie Kanneluren oder Kapitellformen für die Maler und die Betrachter nicht so sehr ins Gewicht fielen, wie der Umstand, dass in dem Fries zahlreiche Konstellationen und Vorgänge erfasst waren, die ihnen aus dem Alltag bzw. von den nundinae bekannt waren. ${ }^{554}$ Letztendlich kann diese Frage nicht beantwortet werden, doch mit Sicherheit würde auch ein besonders spitzfindiger Betrachter feststellen oder dem Eindruck erliegen, dass mit den Statuenpostamenten und Säulenhallen das Dargestellte den Begebenheiten auf dem forum von Pompeji zumindest so sehr ähnelte, dass entsprechende Assoziationen hervorgerufen wurden.

Auf dem Fragment, das in diesem Zusammenhang besonders relevant ist (Abb. F51), sind vor dem üblichen Hintergrund mit zweigeschossiger Säulenstellung vier in toga bzw. Mäntel gehüllte Personen zu sehen, die dem Betrachter den Rücken zukehren. ${ }^{55}$ Sie schauen auf eine weiße Fläche, die direkt unterhalb der Oberkante

548 Vgl. Nappo 1989, 90-92.

549 Vgl. die Abbildungen bei Olivito 2013, 37-83 Abb. 20-62.

550 Vgl. dazu Parslow 1998, 113-114.

551 Vgl. Mau 1908, 51-53; Nappo 1989, 94-95.

552 Am Nordende der Via delle Scuole und amWestende der Via dell'Abbondanza sind auch im Bordstein Spurrillen zu erkennen. In der Via delle Scuole beträgt der Abstand zu dem am Straßenende platzierten Brunnen etwas über $150 \mathrm{~cm}$, sodass ein Fuhrwerk wie der in der Casa del Fabbro (I 10, 7) gefundene Wagen mit einer Breite von $130 \mathrm{~cm}$ theoretisch auch daran hätte vorbeifahren können (Vgl. Allison 2006, 190). Dies deutet darauf hin, dass das forum ungeachtet möglicher Verbote von Fuhrwerken befahren wurde. Geschwächt wird diese These dadurch, dass die Bordsteine an den entsprechenden Stellen aus weichem Tuff bestehen und dass nicht ausgeschlossen werden kann, dass die Spurrillen erst während oder nach der Ausgrabung entstanden. Sumiyo Tsujimura untersucht jedenfalls nur die Spurrillen in den Trassen: Tsujimura 1991, 61 zur Breite der Fuhrwerke.

553 Dazu auch weitere Argumente: Guzzo 2007, 158.

554 Vgl. Olivito 2013, 168. Ähnlich auch Nappo 1989, 94-95.

555 Ausführliche Besprechung dieses Abschnittes mit Bibliographie: Olivito 2013, 68-70. 
der Sockel dreier Reiterstatuen angebracht ist. ${ }^{556}$ Diese weiße Fläche ist mit einem roten Rahmen eingefasst. An den seitlichen Rändern ist die Malerei so beschädigt, dass keine Enden der weißen Fläche zu erkennen sind. Theoretisch wäre es daher möglich, dass sie sich noch weiter fortsetzte. ${ }^{57}$ Auf der Tafel sind in vier Zeilen kleine Linien zu erkennen, die darauf geschriebenen Text andeuten. ${ }^{558}$ Zudem meint Olivito zwei senkrechte Linien erkennen zu können, die eine Gliederung des Textes in drei Kolumnen darstellten. ${ }^{559}$ Aufgrund der geschilderten Merkmale - Anbringungsort an bestehenden Monumenten auf dem forum, weiße Farbe und Beschriftung sowie dem langen, schmalen Format - kann die Tafel als eine tabula dealbata gedeutet werden, wie sie in Schriftquellen erwähnt werden, also eine Holztafel, die weiß angestrichen und mit öffentlichen Ankündigungen oder Mitteilungen beschrieben wurde. ${ }^{560}$

Dass es sich bei der Tafel um eine Darstellung von auf dem forum ausgestellten Texten von öffentlichem Interesse handelt, ist unumstritten. ${ }^{561}$ Was den Inhalt der Texte betrifft, so ist es am wahrscheinlichsten, dass Gesetzestexte oder Verordnungen gemeint sind, die für eine gewisse Zeit für die ganze Einwohnerschaft sichtbar ausgestellt wurden, bevor sie im Stadtrat beschlossen werden sollten. Diese Praxis ist in den Schriftquellen belegt und war vermutlich mit den nundinae verbunden, $\mathrm{zu}$ denen auch die im Umland lebende Bevölkerung in das Stadtzentrum kam. ${ }^{562}$ Über die genaue Gliederung oder den konkreten Inhalt der Texte kann nichts gesagt werden. Es handelt sich bei den „Schriftzeichen“ nicht um tatsächliche Buchstaben, sondern nur um die Andeutung von Schrift. Ob zum Beispiel wie in den Quellen geschildert mit Rot einzelne rubricae abgegrenzt und dann mit Schwarz ausgefüllt wurden, ist nicht zu erkennen. ${ }^{563}$

Relevant ist auch hier eine doppelte Natur der Darstellung: Auf einer bildimmanenten Ebene handelt es sich um einen Text, der von Lesern inhaltlich rezipiert wird. Für den antiken wie den modernen Betrachter des Forumfrieses ist die beschriebene Holztafel als ein Artefakt zu erkennen, das in einem bestimmten zeitlichen und räumlichen Kontext verortet war und charakteristische materielle Eigenschaften hatte. Die tabula ist hier Medium und Artefakt zugleich. Die Darstellung der tabula wird dabei auf wenige und offenbar am meisten charakteristische Merkmale reduziert: die Länge,

556 Die Reiterstatuen entsprechen denen, die auch auf anderen Fragmenten des Frieses zu sehen sind: Olivito 2013, 67 Abb. 45; 77 Abb. 53.

557 Auf einem Stich aus dem Jahr 1762 endet die weiße Fläche jedoch links hinter dem Kopf einer der Figuren, während sie rechts über den Rand des Fragmentes hinauszureichen scheint: (ohne Autor) 1762, Taf. 43 (S. 227, bei Ausrichtung auf den Betrachter rechts unten).

558 Vgl. (ohne Autor) 1762, 226.

559 Zur ausführlichen Beschreibung der Szene vgl. besonders: Olivito 2013, 70.

560 So auch: (ohne Autor) 1762, 226 Anm. 11.

561 Ausführlich dazu: Olivito 2013, 201-206. Vgl. Gesemann 1996, 170; Guzzo 2007, 158; Nappo $1989,88$.

562 Vgl. Olivito 2013, 70; 204-206.

563 Lex Acilia repetundarum (CIL I 583) 14. Liv. 9,46,5; Eck 1998, 211-215; Eck 1999, 58-60; Rea $1977,153$. 
den Rahmen, die weiße Farbe, eine rote und/oder schwarze Beschriftung und ihre Befestigung an den Monumenten. ${ }^{564}$

Die zu den Schriftquellen gestellten Fragen lassen sich anhand dieser Darstellung ebenfalls weitgehend beantworten. Anbringungsort und Material werden explizit thematisiert. Der Inhalt kann nur grob eingegrenzt werden. Die Rezeption des Textes bzw. der tabula dealbata wird vor allem in der bildimmanenten, inhaltlichen und artefaktischen Ebene reflektiert. Versteht man diesen Bildausschnitt im Zusammenhang des gesamten Frieses, wird deutlich, dass das Lesen solcher Bekanntmachungen als eine der typischen, auf dem forum und besonders bei den nundinae stattfindenden Tätigkeiten aufgefasst wurde.

Als Akteure sind hier nicht die Schreiber, sondern lediglich die Leser wiedergegeben. Der Personenkreis der dargestellten Leser ist anhand ihrer Kleidung und Körpergröße als heterogen dargestellt. Alle sind männlich. Drei von ihnen tragen bräunliche Mäntel oder togae, einer eine weiße. Auch die Schuhe sind verschieden. Die ganz rechts stehende Figur ist kleiner als die anderen, sodass hier ein Kind oder Jugendlicher gemeint sein könnte. Alle vier stehen ruhig und haben den Körper ganz den Texten zugewandt, halten jedoch genug Abstand, um größere Abschnitte der Tafel überblicken zu können. Hier handelt es sich um ein intensives und konzentriertes Lesen in ruhender Haltung. ${ }^{565}$ Dies ist durch die kleinen Buchstaben und die Gliederung des Textes vorgegeben und stellt einen markanten Unterschied zu den programmata und edicta munerum dar, deren Hauptbestandteile auch bei schneller Bewegung zu lesen waren.

Der Anbringungsort an den Statuensockeln verdeutlicht noch einen weiteren Aspekt: Die Tafel ist im Bild an der Vorderseite der Sockel der Reiterstatuen angebracht. Bei den erhaltenen Sockeln an der Westseite des forum von Pompeji befinden sich die Ehreninschriften wie üblich genau dort. Somit sind die Ehreninschriften durch die tabulae komplett verdeckt. Indem diese nun nicht mehr zu sehen sind, findet eine Hierarchisierung der zwei Inschriftengattungen statt. Die Belange der colonia wurden - wenigstens zeitweise - über das Bedürfnis der Individuen nach öffentlicher Repräsentation gestellt und ihre Monumente gewissermaßen zum Mobiliar des forum degradiert.

564 Auf die Lesbarkeit wie z. B. in der Darstellung des Menander, wurde dagegen verzichtet. Siehe: CIL IV 7350b. Vgl. Parise Badoni 1990, 367 Abb. 204; Ling/Ling 2005, 87.

565 Zurecht bemerkt Olivito, dass sich solche Leseszenen überall und alltäglich im römischen Reich ereignet haben müssen und dass es erstaunlich ist, dass die Darstellung in der römischen Kunst ohne Parallelen ist: Olivito 2013, 72. Dies kann zumindest zum Teil daran liegen, dass gerade solche „Nachrichtenanschläge“ in der Regel tatsächlich vor allem in ihrem medialen Charakter wirkten und möglicherweise einen allzu alltäglichen Anblick boten. 


\subsection{Zwischenergebnis}

Insgesamt betrachtet werden in den Schriftquellen und den bildlichen Darstellung unterschiedliche Aspekte der Produktion und Rezeption von Steininschriften, Dipinti und Graffiti aufgegriffen. Die beteiligten Akteure, mögliche Anbringungsorte, Reaktionen von Lesern und Inhalte werden teilweise sehr genau angegeben und stehen oft in kausalem Verhältnis zur übergeordneten Handlung. Der Vorgang der Anbringung sowie die konkrete Gestaltung, also zum Beispiel die verwendete Schriftart, werden dagegen selten thematisiert.

In den als Metatexte vorgestellten Inschriften bzw. Inschriftengruppen, der Inschrift für Marcus Nonius Balbus sowie den Epigrammen und Signaturen, werden weitere Bezüge auf Bedeutungszuschreibungen an Inschriften greifbar. Die Texte verweisen sowohl auf sich selbst als auch auf andere Inschriften derselben Gattung. Doch wo der Graffito den Charakter eines ironischen Kommentars hat, der vor allem mögliche kritische Reaktionen vorwegnimmt, ist die Inschrift am Altar des Nonius Balbus dezidiert normativ. Durch offizielle Formeln aber auch durch genaue Angaben der Begleitumstände werden Ort, Kontext und Inhalt der Inschriften festgeschrieben. Es wird deutlich, dass die Inschrift keine Beischrift zum Monument, sondern selbst Teil des Monuments ist.

Die vorgestellten Bilder beinhalten Schriftzeugnisse, die inhaltlich und formal eng mit dem jeweiligen Kontext verknüpft sind. Die tabulae dealbatae etwa mussten notwendigerweise im Zentrum des öffentlichen Lebens aufgestellt werden. In beiden Bildern wird darüber hinaus deutlich, dass die Schriftzeugnisse als wichtige oder emblematische Bestandteile dieser Räume gesehen wurden. Auch hier kann beobachtet werden, dass die Familie der Satrii offenbar erfolgreich darin waren, den eigenen Namen im öffentlichen Raum zu präsentieren und damit bestimmte Teilräume zu beanspruchen.

Fasst man die Ergebnisse dieser Auseinandersetzung mit den Metatexten zu Inschriften im öffentlichen Raum zusammen, stellt sich die Frage, inwieweit diese neue Aufschlüsse bieten können, die durch die Beschäftigung mit dem Material selbst nicht möglich waren. Als wichtigster Punkt ist die explizite Verknüpfung mit dem räumlichen Kontext zu nennen. Diese scheint zwar selbstverständlich daraus hervorzugehen, dass die tatsächlich gefundenen Inschriften immer automatisch an einen Punkt im Raum gebunden waren. Gerade der heterogene Erhaltungszustand macht es jedoch an manchen Orten schwierig, zufällige Häufungen auszuschließen. Die Schriftquellen und die gezeigten Bilder verdeutlichen dagegen, dass bestimmte Teilräume als Orte wahrgenommen wurden, an denen man Graffiti finden oder schreiben konnte, an denen bestimmte Inschriften aufzustellen waren und an denen Inschriften Teil der Fassadengestaltung waren. Als zweiter wichtiger Punkt erweisen sich die in den Texten bezeugten Rezeptionspraktiken. Besonders bei Wandinschriften ist es unklar, inwieweit sie über die manchmal nur kurzfristig aktuellen Inhalte hinaus relevant waren. Hier können die vorgestellten Quellen zeigen, dass weitere Rezeptionsfor- 
men neben dem Lesen vorhanden waren. Gerade das Lesen nämlich wird nicht explizit thematisiert, wohl aber diesem vorangehende und nachfolgende Handlungen. Es zeigt sich jedoch auch, dass die Beurteilung der Wandinschriften je nach Anlass variierte. Insofern bieten die Schriftquellen, Inschriften, die sich auf andere Inschriften beziehen, und Bilder, die Schriftzeugnisse beinhalten, einen Einblick über die Voraussetzungen, mögliche Funktionen und die Wahrnehmung von Schrift im öffentlichen Raum. 
